\title{
XXIV.
}

Mitteilung aus der I. chirurgischen Klinik der königl. ungarischen Universität zu Budapest. (Vorstand: Prof. Dr. Jul. Dollinger.)

\section{Beiträge zur Krehsstatistik, mit besonderer Berücksichtigung der an der I. chirurg. Universitätsklinik durch operative Behandlung erzielten Danererfolge. $\left.{ }^{1}\right)$ \\ Von}

Dr. Paul Steiner,

Operationszögling der Klinik.

Seit der Gründung des „Komitee für Krebsforschung“ in Deutschland begann eine Strömung in den Fachkreisen zur Erforschung der Verbreitung der Krebskrankheit. Die statistischen Ausweise der verschiedenen Länder liefern den Beweis, wie zahlreich der Krebs vorkommt und wir stehen unter dem Eindrucke, daß die Krebskrankheit in Zunahme begriffen ist. Jedoch können wir diese Annahme nicht als gerechtfertigt betrachten, denn mit Hilfe neuerer Untersuchungsmethoden diagnostizieren wir in vielen solchen Fällen den Krebs, in welchen wir bis jetzt die pathologische Veränderung für gutartige oder andere bösartige Neubildung hielten.

Jede Bewegung bezweckt die Frühdiagnose des Krebses. Boas verlangt die Einrichtung von Untersuchungs-Stationen für Krebse in den großen Zentren, in denen durch verfeinerte diagnostische Technik die Diagnose in allen krebsverdächtigen Fällen so früh als möglich gestellt werden soll. Mayo Robson befürwortet die Beteiligung des Publikums an der Bewegung; anch Witheford erblickt den Erfolg im Kampfe gegen den Krebs in der Aufklärung des

1) Dollinger, Die Dauererfolge der operativen Behandlung des Krebses in der I. chirurgischen Universitätsklinik in Budapest. Deutsche med. Wochenschrift 1905, Nr. 37. 
Volkes, auch Kocher ruft die Ärzte in seiner auf dem I. internationalen Chirurgen-Kongreß gehaltenen Eröffnungsrede zum Kampfe gegen den Krebs auf.

In Ungarn regte Prof. Dollinger, als Vorstand des KrebsKomitees der kgl. Ärztegesellschaft, zur Sammlung krebsstatistischer Daten an. Er sucht nicht nur die Zahl der Krebskranken, sondern er richtet seinen Blick auch auf den Zusammenhang der Krebskrankheit von Menschen und Tieren, was vom ätiologischen Standpunkte aus wichtig ist. Auch ist er bestrebt, den weiteren Verlauf der operierten Fälle, den Zeitpunkt der Rezidive festzustellen, was für den Wert der chirurgischen Behandlung entscheidend ist.

Schon Volkmann verwirft den Standpunkt des „noli me tangere" und befaßt sich im Jahre 1875 eingehend mit der Frage der Rezidive. Billroth forscht im Jahre 1880 nach der Zeit der Rezidive, und widerlegt die damalige Auffassung des ,quanto magis palpatur, magis indignatur." Volkmann beginnt erst nach Ablauf eines Jahres zu hoffen, daß sich keine Rezidive einstellen und spricht erst nach 3 Jahren von "definitiver Heilung."

Winter rechnet auf dem gynäkologischen Kongreß 19015 Jahre als Maß der definitiven Heilung, wobei zu betonen ist, daß auch nach 3 resp. 5 Jahren noch Rezidive auftreten können, welche wir als „Spätrezidive“ bezeichnen.

Da der Wert der chirurgischen Behandlung nach dem Auftreten der Rezidive beurteilt wird, ist es notwendig, den Begriff der Rezidive kurz zu erörtern. Wodurch entstehen also die Rezidive?

1. Aus den zur ückgebliebenen Teilen der Geschwulst. Diese Fntstehungsart wird bei der radikalen Richtung der modernen Chirurgie stets seltener, und begegnen wir ihr doch, so finden wir dieselbe nach kurzer Zeit.

2. Aus den Krebszellen des Lymphapparates. Die Krebszellen haben nicht nur in den Lymphdrüsen, sondern anch in den Lymphwegen ihren Sitz.

3. Durch Inokulation (Impfrezidive). Die Möglichkeit dieser Verbreitungsart erwähnen anch Borst und Kahlden, und Levesque berichtet über einige Fälle der Inokulations-Rezidive. Diese Entstehungsart gehört zu den selteneren und die diesbezüglichen Versuche Thorns bei 6 Fällen von inoperablem Carcinom ergaben ein negatives Resultat. Deswegen ist auch die Annahme dieser Verbreitungsart nur in jenen Fällen gerechtfertigt, bei denen die Möglichkeit der Verbreitung der Krebszellen durch den Lymphapparat ausgeschlossen ist. 
4. Kahlden erwähnt die multizentrische Entwicklung des Krebses, d. h. der Krebs entsteht auf mehreren von einander unabhängigen Stellen. Petersen berichtete auf dem chirurgischen Kongreß 1901 von mehreren derartigen auf die Haut lokalisierten Krebsen und auch wir hatten die Gelegenheit, mehrere Fälle von „Carcinoma multiplex" zu beobachten. Die multizentrische Entwicklung entsteht nicht unbedingt zu gleicher Zeit, sondern auch "pluritemporal" und so kann es sich ereignen, daß der Krebs nach Entfernung eines Knotens, von diesem unabhängig, nicht als Rezidiv im engeren Sinne des Wortes, in solchen Zellen auftritt, welche zur Zeit der Operation noch vollkommen gesund oder schon anaplastisch waren.

Kahlden erklärt die Spätrezidive durch plurizentrale, pluritemporale Entwicklung, König beantwortet die Frage mit seiner Schlummerzellentheorie, Thiersch, Bruns, Tillmanns nehmen ganz neue Veränderungen an, Rindfleisch erblickt die Ursache in der Fortpflanzung des formativen Reizes.

Jordan referierte auf dem 33 . Kongreß der deutschen Gesellschaft für Chirurgie über 2 Spätrezidivfälle, der eine trat nach 19 Jahren bei einem Zungenkrebs, der andere nach 15 Jahren bei einem Brustdrüsenkrebs auf. Auch wir beobachteten unlängst bei einer 55jährigen Fran, welche vom Prof. Kovács vor 13 Jahren an Brustdrüsenkrebs operiert wurde, Spätrezidive. Das erste Rezidiv stellte sich nach 4 Jahren, das zweite nach 9 Jahren ein.

Wie auch der Krebs und die Rezidive entstehen mögen, in praktischer Hinsicht muß der Krebs nach Léjars als Infektionskrankheit aufgefaßt werden. Und dies mit Recht. Frühdiagnose, radikale en bloc Operation ist die Devise der jetzigen Chirurgie!

Zur Beurteilung dessen, was wir durch die chirurgische Behandlung des Krebses erreichen können, ließ Prof. Dollinger an alle Kranken, die wir auf der Klinik vom 1. September 1897 bis zum Jahre 1. September 1901 operierten, Fragebogen mit folgenden Fragen versenden:

1. Wie befinden Sie sich derzeit?

2. Ist anf der operierten Stelle eine neue Geschwulst oder ein Geschwür entstanden, wenn ja, wann?

3. Ist seit der Operation an irgend einer anderen Körperstelle eine Geschwulst oder ein Geschwür entstanden?

4. Wurden Sie seit Ihrem Abgang von der Klinik operiert, wenn ja, wo nnd wann? 
War die Antwort des Kranken nicht befriedigend, wandten wir uns um neue Aufklärung an ihn, oder aber nannte er seinen Arzt, ersuchten wir denselben um Bescheid. Ist unser Brief mit der Bemerkung "gestorben“ zurückgestellt worden, wandten wir uns an die entsprechende Behörde zur Mitteilung der Ursache und Zeit des Todes. Manche Ämter wiesen uns an die Angehörigen des Kranken und so gelang es uns auch die Art und die Zeit des Auftretens der Rezidive zu erfahren.

Von den eingelangten Antworten und persönlich festgestellten Befunden sind wir bei dieser Arbeit ausgegangen.

Da die Nachforschung und der Briefwechsel bis 1. Septbr. 1904 fortdanerte, waren für die 3jährige Rezidivfreiheit, d. i. für die Beurteilung der definitiven Heilung nach Volkmann, alle jene Fälle zu verwerten, die wir vom 1. Sept. 1897 bis zum 1. Sept. 1901 d. h, innerhalb 4 Jahren operierten. Während dieser Zeit machten wir 226 Radikaloperationen und es wurde uns der weitere Verlauf von 175 Fällen, d. h. von 77,3 Proz. bekannt. Wenn wir im Sinne von Winter eine 5jährige Rezidivfreiheit fordern, waren nur die operierten Fälle vom 1. Sept. 1897 bis 1. Sept. 1899, d. h. ein zweijähriges Material verwertbar. In diesem Zeitraum wurden 109 radikale Operationen ausgeführt, in 80 Fällen, d. h. bei 73,39 Proz., ist uns der weitere Verlanf bekannt.

Es ist noch die Frage zu beantworten, was uns als Grundlage für die Beantwortung der Rezidivfreiheit dienen soll. Allgemein ist es ein angenommener Standpunkt, daß bei Beurteilung der Frage der Rezidivfreiheit in Abrechnung zu ziehen sind:

1. Die operative Mortalität.

2. Die an einer interkurrenten, nicht krebsartigen Krankheit gestorben sind.

3. Deren weiteres Los uns unbekannt ist.

Gegen diese Berechnung lassen sich folgende Einwendungen machen:

1. Die Kranken, die an operativen Komplikationen gestorben sind, sind indirekt doch infolge des Krebsleidens zugrunde gegangen. Die Patienten, die den operativen Komplikationen zum Opfer fallen, gehören auf das Verlustkonto der chirurgischen Behandlung, dürfen daher bei der Bilanz nicht einfach gestrichen werden.

2. Eine gewisse Zahl jener, die an einer interkurrenten Krankheit gestorben sind, ist mit großer Wahrscheinlichkeit das Opfer ihrer Krebskrankheit geworden. Die im Totenschein angegebene 
Diagnose ist durch die Sektion nicht bewiesen worden, daher glauben wir uns weniger zu irren, wenn wir diese Fälle von der Grundsumme, auf die wir die Prozente der Dauererfolge berechnen, nicht abziehen.

3. Bei der Berechnung ist die Zahl jener in Abzug zu bringen, deren weiteres Los uns unbekannt ist, da wir über ihr weiteres Schicksal gar keine sicheren Anhaltspunkte haben.

In diesem Sinne haben wir bei der Berechnung der Dauererfolge von der Gesamtsumme der Operierten alle Fälle weggelassen, deren weiteres Los uns unbekannt ist, und unsere Berechnung basiert auf jene Fälle, deren weiteres Schicksal wir kennen.

Zur Charakterisierung unseres Materials muß noch bemerkt werden, daß es sich auf die Kranken der Universitätsklinik No. I beschränkt, deren sämtliche Krankengeschichten in den Jahrbüchern der Klinik veröffentlicht sind. Von der Aufnahme auf die Klinik wurden nur die Altersschwachen, die Kachektischen und diejenigen Patienten ausgeschlossen, bei denen man nicht mehr hoffen konnte, daß es gelingen wird, die Krebsgeschwulst grïndlich zu entfernen. Das Material enthält die von Prof. Dollinger, sowie von seinen Assistenten und Operationszöglingen operierten Fälle; es ist also bezüglich des Operateurs nicht einheitlich.

Wie wir sehen werden, sind die Erfolge der Klinik, wie es aus den nachfolgenden 11 Abschnitten erhellt, gut und anregend und rechtfertigen den Ausspruch Virchows: "Ist der Krebs in seinem Beginn und oft sehr lange ein örtliches Leiden, so muß es auch möglich sein, ihn in dieser Zeit örtlich zu heilen!"

\section{Der Gesichtskrebs.}

Der Hautkrebs des Gesichts ist unter den Carcinomen des Organismus ziemlich hänfig und bildet nach Gurlt 1/7, nach Heimann $1 / 10$ sämtlicher Krebse. Am häufigsten erkrankt die Unterlippe. Den Unterlippenkrebs behandeln wir wegen der großen Zahl dieser Fälle in einem speziellen Abschnitt. Dann, wie wir es aus der Bergmannschen Statistik ersehen, ist der Nasenkrebs am häufigsten. Schon in weitaus geringerer Zahl treffen wir die Erkrankung der Gesichtshaut, der Augenlider, der Oberlippe und des Kinns an. Die verschiedenen Formen der Hantkrebse, wie das in der Fläche fortschreitende Ulcus rodens, und den in die Tiefe wuchernden und auf der Oberfläche papillär hervorragenden Krebs konnten wir öfters beobachten. Diese Arten der Hautkrebse sind 
nicht scharf zu begrenzen, da wir auch oft Übergangsformen sehen.

Mit Gesichtskrebs lagen im ganzen 33 Kranke auf der Klinik: 22 Männer, 11 Frauen. Ihr Alter schwankte zwischen 18-68 Jahren. Bei einer 18jährigen zerstörte der auf der Nasenspitze seit 6 Jahren bestehende Tumor die Nasenflügel und den knorpeligen Teil des Septum. Bei einem 26jährigen zerstörte der seit 3 Jahren bestehende Krebs den linken Nasenflügel. Bei einem 35jährigen Mann begann der Krebs vor 11 Jahren, folglich in seinem 24. Jahre, und bei der Aufnahme lag eine talergroße Geschwulst vor.

Nach Alter gruppieren sich unsere Fälle folgendermaßen:

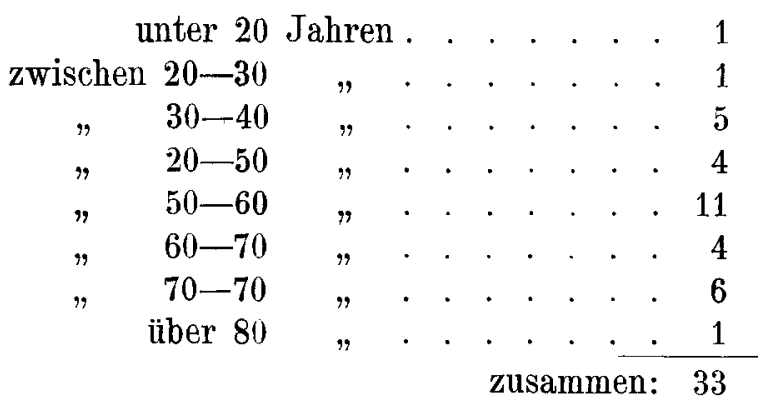

Wir sehen also, daß sich die überwiegende Zahl zwischen 50-60 Jahren und zwar derartig verteilt, daß zwischen 50-55 Jahren 7, zwischen 55-60 Jahren 4 Kranke zur Aufnahme gelangten.

Was den Sitz des Krebses betrifft, begann der Krebs in 4 Fällen auf den Augenlidern, in 10 Fällen auf der Nase, in 14 Fällen auf der Gesichts- und Schläfenhaut, in 4 Fällen auf der Ohrenmuschel und in 1 Falle im Genick. Die Größe der Geschwulst wechselte zwischen einem Knötchen und der Größe eines Apfels, überwiegend waren die exulcerierten Fälle. Die Geschwüre waren teils flach mit aufgeworfenen Rändern, teils waren sie kraterartig vertieft und in manchen Fällen beschränkten sie sich nicht nur auf die Gesichtshaut, sondern zerstörten auch die benachbarten Teile. Diese Geschwüre variierten von Kronen- bis zur Handgröße und wir hatten Fälle, wo die krebsartige Zerstörung sogar auf die knochigen Ränder des Sinus pyriformis, ferner durch die Nasenhöhle fort. schreitend auf den Gaumen, weiter auf die Augenhöhle übergriff. Bei ausgedehnten Krebsen, bei längere Zeit bestehenden Fällen, so in einem Fall 14 Jahre nach dem Beginn des Leidens waren die regionären Drüsen nicht immer krebsig infiltriert, was ein Beweis für die relative Gutartigkeit dieser Krebsart ist. 
Es ist allgemein bekannt, daß der oberflächliche Gesichtskrebs zur spontanen narbigen Schrumpfung neigt, dies kann aber nicht als definitive Heilung angesehen werden. Bergmann machte öfter die Erfahrung, daß an den Rändern der Narbe neuere kleine Geschwüre entstehen, daß in der Narbe Krebszellen selbst mikroskopisch nicht nachweisbar waren und der Krebs in den regionären Lymphdrüsen sich fortpflanzte. Deswegen können wir die durch die Röntgentherapie oder durch Radium erzielten Erfolge vorläufig noch nicht als definitive betrachten. Prof. Dollinger meint in einem seiner Vorträge: „Bei den meisten Krebsen sind die regionären Lymphdrüsen bereits infiltriert, der Kranke hat folglich außer der sichtbaren und fühlbaren primären Geschwulst noch mehrere unsichtbare Krebsherde in den regionären Lymphknoten. Wollen wir den Kranken heilen, so müssen wir alle verdächtigen regionären Drüsen gründlichst entfernen. Die Röntgen- und die Radiumtherapie bilden daher vorläufig noch keinen sicheren Ersatz der chirurgischen Behandlung."

\section{A. Primärkrebse.}

Zur Beurteilung der operativen Erfolge, wenn wir unsere Statistik nach Volkmanns Triennium aufstellen, verfügen wir über 15 Fälle primären Gesichtskrebses, von denen uns bei 13 der weitere Verlauf bekannt ist; in 5 Fällen zeigten sich innerhalb 3 Jahren keine Rezidive, in diesen 5 Fällen verliefen zwischen Beginn des Krebses und der Operation in 2 Fällen $1 \frac{1 / 4}{4}$ resp. 11/2 Jahre, in 3 Fällen sogar 6 Jahre. In 8 Fällen zeigten sich Rezidive; in diesen Fällen verstrichen 7 Monate bis 20 Jahre von dem Beginne der Krankheit bis zur Zeit der Operation. Die Rezidive stellten sich nach $1 / 2-2 \frac{1}{2}$ Jahren ein. Daß wir verhältnismäßig eine größere Zahl von Rezidiven hatten, begründen wir mit der Häufigkeit „schwerer Fälle“, mit der Ausdehnung des Krebses. Damals wurden nur fortgeschrittene Krebsfälle auf der Klinik aufgenommen, die oberflächlichen Fälle operierten wir ambulatorisch, daher wurden sie in unserer Statistik nicht berücksichtigt.

Von den 8 Rezidivfällen starben bereits 6 infolge des Krebses, 2 leben mit dem Rezidiv. Bei letzteren stellte sich die Rezidive nach 4 resp. 6 Jahren ein.

Berechnen wir die operative Statistik des Gesichtskrebses in Prozenten und gründen wir unsere Berechnung auf die bekannten 13 Fälle, so erhalten wir folgendes Resultat: 


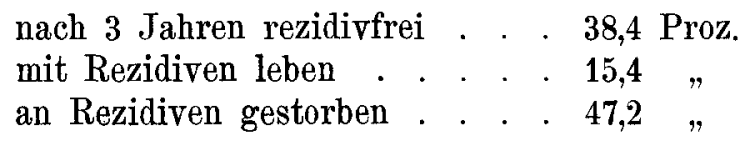

Nach der Statistik von Batzaroff stellten sich auf der Züricher Klinik nach 175 Gesichtskrebs-Operationen in 66 Fällen (37,7 Proz.) Rezidive ein, eine Rezidivfreiheit wurde in 62,3 Proz. erzielt; auf der Würzburger Klinik nach der Statistik von Stöckler nach 72 Operationen stellten sich 32 (44,4 Proz.) Rezidive ein, eine Rezidivfreiheit wurde in 55,6 Proz. erzielt. Wenn wir verhältnismäßig mehr Rezidive hatten, müssen wir wiederholt auf den Umstand hinweisen, daß bei uns nur die schwersten Fälle klinisch behandelt wurden.

Nehmen wir als Maß der definitiven Heilung die auf dem Gießener Gynäkologen-Kongreß von Winter vorgeschlagenen 5 Jahre, so ist die Zahl unserer Fälle 10; das weitere Los ist bei 8 Fällen bekannt. Von diesen sind rezidivfrei nach 5 Jahren 3 , mit Rezidiv lebt 1, infolge der Rezidive starben 4. Prozentual ausgedrückt und das Resultat anf die uns bekannten 8 Fälle bezogen, sind 37,5 Proz. rezidivfrei nach 5 Jahren.

\section{B. Rezidivkrebse.}

Wir operierten in 8 Fällen rezidive Gesichtskrebse; bei 5 von denselben traten die Rezidive nach einer auf der Klinik, bei 3 nach anderswo gemachten Operationen auf. Die Rezidive waren von großer Ausdehnung, so erstreckte sich in einem Falle der vom Augenlid ausgehende Gesichtskrebs bis in die Orbita, der Bulbus war bereits anderswo enukleiert und die Augenhöhle war bei der Aufnahme von Krebs ausgefüllt. In einem anderen Falle zerstörte der von der Ohrmuschel ausgehende Krebs das Periost der Schläfe, die Trommelhöhle und die Geschwulst verbreitete sich sogar auf die Dura mater. Von den 8 operierten Fällen ist uns das Los von 7 bekannt und zwar:

$$
\begin{aligned}
& \text { nach } 3 \text { Jahren rezidivfrei . . . . . } 2 \\
& \text { an Rezidiven gestorben . . . . . . } 5
\end{aligned}
$$

In den rezidivfreien Fällen bestanden die Rezidive, die eine neue Operation erforderten, seit 1 resp. 2 Monaten; in den rezidivierten Fällen hingegen seit 10 Tagen, 2-3 Wochen, 11/2-4 Monaten. Die zwischen der Rezidivoperation und der folgenden Rezidive verlaufene Zeit war von 2 Wochen bis 6 Monaten. In einem Falle, wo der Krebs auf der rechten Schläfe saß, sahen wir ein vier- 
maliges Rezidiv, das sowohl lokal, wie regionär in 2 wöchentlicher Zwischenzeit auftrat. Unter den regionären Metastasen war eine in der Fossa supraclavicularis sitzende mit der Vena subclavia verwachsene größere Drüsenmetastase.

Bei zwei Rezidivoperationen bekameu wir keine neuerliche Rezidive. Der eine Fall betraf eine 66jährige Frau, bei der zur Zeit der ersten Operation die den rechten Nasenflügel zerstörende Geschwulst schon seit 6 Jahren bestand, die Rezidive traten nach 2 Monaten auf, und gingen sogar auf die Nasenhöhle über. Nach der radikalen Entfernung der Rezidive ist die Frau schon 6 Jahre rezidivfrei. Der andere Fall kam bei einer 18jährigen Frau vor, bei der die Nasenflügel und die knorpelige Nasenscheidewand zerstört waren, die Rezidive stellten sich nach Verlauf eines Monats ein, nach der Entfernung der Rezidivgeschwulst erzielten wir eine 6jährige Rezidivfreiheit.

Wenn wir unsere Statistik in Prozenten ausdrücken, so erhalten wir anf die 7 Fälle berechnet:

rezidivfrei nach 3 Jahren . . . 28,6 Proz.

an Rezidiven gestorben . . . 71,4 "

Berechnen wir die Statistik auf 5 Jahre, so verfügen wir über 6 Fälle, von denen der Verlauf von 5 bekannt ist und zwar:

rezidivfrei nach 5 Jahren . . . . . . 2

an Rezidiven gestorben . . . . . . 3

oder in Prozenten ausgedrïckt, beträgt die definitive Heilung 40 Proz.

\section{Der Lippenkrebs.}

Unter den Gesichtskrebsen ist der Lippenkrebs der häufigste. Trendelenburg hatte unter 499 Gesichtskrebsen 241 (48,3 Proz.) Lippenkrebse. Die Unterlippe ist bekanntlich häufiger der Sitz des Krebses, als die Oberlippe. In unseren Fällen saß der Krebs

auf der Unterlippe in . . . . . 84 Fällen auf der Oberlippe in . . . . 6 " im Mundwinkel in . . . . . . 9 " auf der Ober- u. Unterlippe in . 1 Falle unbekannt in . . . . .... 4 Fällen zusammen 104 Fälle.

d. h. 6,6 Proz. der Lippenkrebse saß auf der Oberlippe, nach Bruns 5,5 Proz., nach Wölfler 4 Proz. Nach Fricke und Loos ist der 
Oberlippenkrebs $17 \mathrm{mal}$, nach Wörner $18 \mathrm{mal}$ seltener als der Unterlippenkrebs, nach unserer Statistik 15,5mal seltener.

Was das Geschlecht betrifft, kam das Leiden in 101 Fällen bei Männern, in 3 Fällen bei Frauen vor, d. h. in 2,89 Proz. der Fälle kam der Lippenkrebs bei Frauen vor. Nach Wölfler in 7,1 Proz., nach Bruns in 7,2 Proz., nach Trendelenburg in 7 Proz., nach Winiwarter in 1,6 Proz. Auffallend ist in unserer Statistik, daß der Oberlippenkrebs in allen 6 Fällen bei Männern vorkam, obwohl allgemein so auch von Fricke geglaubt wird, daß die Oberlippe häufiger bei Frauen erkrankt. Wölfler hatte 8 Fälle von Oberlippenkrebs, unter diesen 5 bei Frauen.

Die Disposition für den Krebs wächst mit dem Alter und zeigt sich nach Trendelenburg am häufigsten zwischen 40 bis 60 Jahren. Auch unsere Fälle beweisen dies, was auch aus folgender Statistik ersichtlich ist:

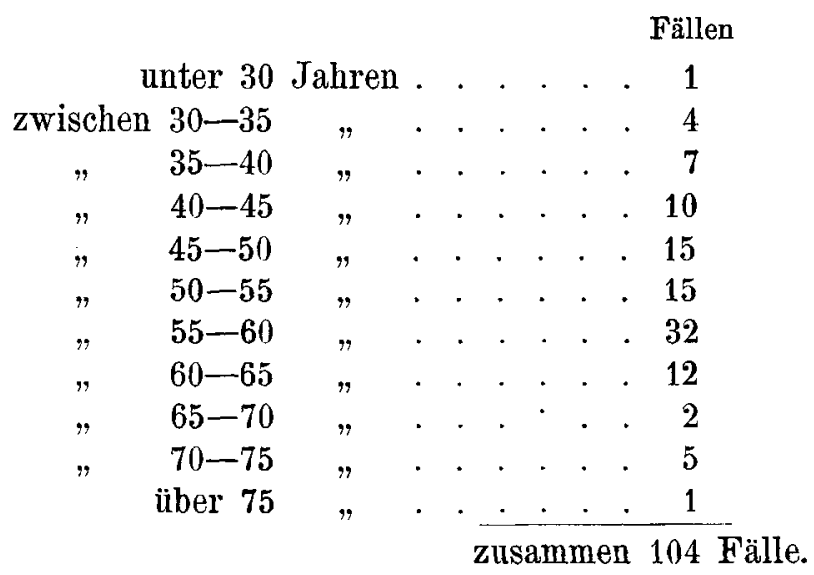

Wir sehen daher, daß der Lippenkrebs am häufigsten zwischen 55-60 Jahren vorkommt, das gleiche fanden Fricke und Wölfler. Nach Winiwarter ist das Vorkommen zwischen 45-50 Jahren, nach Heimann und Thiersch zwischen 50-60 Jahren, nach Bruns zwischen 60-70 Jahren am häufigsten; als Durchschnittsalter kann das 60. Jahr angenommen werden. Die Kranken suchen, von der Wahrnehmung der Krankheit gerechnet, durchschnittlich zwischen dem 5.-12. Monate unsere Klinik auf. Nur ungefähr 63 Proz. kommen gleich im 1. Jahre, die übrigen zeigen sich alle später, nach Wölfler zwischen 5-15 Jahren 5 Proz. Bei uns meldeten sich innerhalb der ersten 6 Wochen 3 Kranke, in 
2 Fällen führten sie die Entstehung des Leidens auf 7 Jahre zurück. Die Fälle geordnet, meldeten sich

$$
\begin{aligned}
& \text { innerhalb } 6 \text { Wochen . . } 3 \text { Kranke } \\
& \text { innerhalb } 1 \frac{1}{2}-3 \text { Monate . . } 15 \text {, }
\end{aligned}
$$

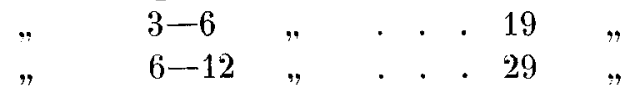

$$
\begin{aligned}
& \text { d. h. im ersten Jahre . . . . } 66 \text { Kranke } \\
& \text { innerhalb 1-2 Jahre. . . . } 6 \text {, }
\end{aligned}
$$

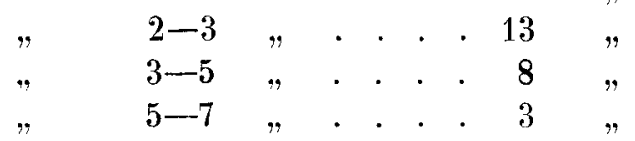

Was die Anamnese anbelangt, machen wir häufig die Beobachtung, daß der Kranke ein starker Raucher, am meisten Pfeifenraucher ist, oder viel Tabak kaut. Schon Sömmering (1795) schreibt dem Tabakgenuß eine große Rolle zu. Wir treffen zwar auch Kranke, die Nichtraucher sind, unter unseren Fällen - die Frauen nicht gerechnet - sind nur 3 Nichtraucher.

Wir machen häufig die Beobachtung, daß die Kranken den an ihrer Lippe entstandenen Knoten, auf ein Trauma zurückführen, doch schreibt die Wissenschaft eine viel größere Bedeutung dem konstanten, oft wiederkehrenden Reize zu. So hielt der Kranke in einem Falle seine Pfeife fortwährend im rechten, in einem anderen Falle im linken Mundwinkel und bei beiden Kranken entstand der Krebs auf der entsprechenden Seite der Lippe.

Oft bilden angeborene und angeeignete Anomalien, gutartige Warzen den Ausgangspunkt des Krebses. So ging der Krebs in einem Falle von einer kleinen, im rechten Mundwinkel schon seit 8 Jahren sitzenden kleinen Warze aus, in einem anderen Falle stellte er sich in einer Bißnarbe ein.

Bei den meisten Fällen war die Geschwulst bereits exulzeriert, die regionären Drüsen vergrößert, in einigen Fällen verbreitete sich der Tumor schon auf die Schleimhaut der Wange. Unser operatives Verfahren besteht darin, daß die regionären Drüsen in allen Fällen durch den Z-Schnitt nach Dollinger aufgesucht und entfernt werden. Wir begnügen uns mit dem Kocherschen Gurgelschnitt nicht, da wir bei einigen unserer Fälle, wo wir sowohl in der submentalen Gegend, wie längs des Kopfnickers die Drüsen entfernt haben, die Erfahrung gemacht haben, daß die Rezidive in den tiefen um die Carotis gelegenen Drüsen auftraten. Daher führen wir an dem lateralen Ende des Gurgelschnittes am inneren 
Rande des Kopfnickers einen weiteren Schnitt bis zur Articulatio sterno-clavicularis aus und setzen ihn längs des Schlüsselbeines fort. Auf diese Weise kann die ganze Halsgegend als ein anatomisches Präparat überblickt und sämtliche Drüsen entfernt werden. Erst nachher wird die primäre Geschwulst fortgeschafft.

a) Primärer Lippenkrebs.

Von den primär operierten Lippenkrebsen kommen, die dreijährige Rezidivfreiheit als Maß angenommen, 56 Fälle in Rechnung, von welchen das weitere Schicksal von 44 bekannt ist.

Es ist hervorzuheben, daß wir keine operative Mortalität hatten. Loos zeichnet 0,4 Proz., Fricke 6,5 Proz. Mortalität auf.

Von den 44 bekannten Fällen sind

$$
\begin{aligned}
& \text { rezidivfrei nach } 3 \text { Jahren . . . . } 31 \text { Fälle } \\
& \text { an Rezidiven gestorben. . . . . } 13 \text { " }
\end{aligned}
$$

In den rezidivfreien Fällen variierte die zwischen dem Bestehen des Krebses und der Operation verflossene Zeit zwischen 6 Wochen und 4 Jahren nach folgender Verteilung:

$$
\begin{aligned}
& \text { innerhalb } 3 \text { Monate meldeten sich . . } 9 \text { Fälle }
\end{aligned}
$$

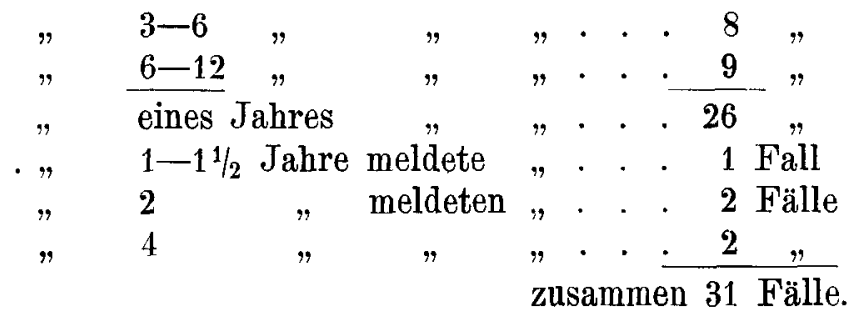

In den rezidivierten Fällen variierte die zwischen dem Bestehen des Krebses und der Operation verflossene Zeit zwischen 6 Wochen und einem 1 Jahr nach folgender Verteilung:

$$
\begin{aligned}
& \text { innerhalb } 3 \text { Monate meldeten sich . . . } 4 \text { Fälle } \\
& \begin{array}{llllllllll}
" & 3-6 & & & & & & & &
\end{array}
\end{aligned}
$$

Der Zeitpunkt der Rezidive variierte zwischen 2 Monaten und 2 Jahren, Die Rezidive stellten sich ein: 


$$
\begin{aligned}
& \text { nach } 2 \text { Monaten bei . . . . } 1 \text { Falle }
\end{aligned}
$$

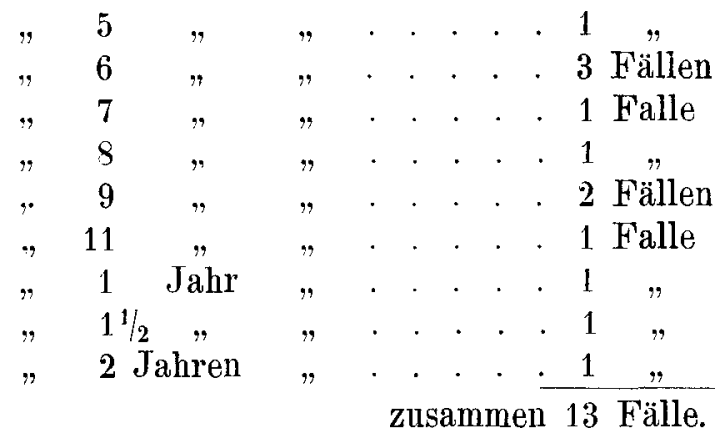

Unter den rezidivierten Fällen befinden sich schon die Lippenkrebse von größerer Ausdehnung, Fälle, bei welchen der Krebs nicht nur auf den Mundwinkel, sondern auch schon auf die Wange überschritt und bereits in ziemlicher Größe exulzeriert war, die ganze Lippe zerstört hat und die submaxillaren Drüsen nicht nur krebsig infiltriert, sondern auch schon exulzeriert waren. Die Rezidive, soweit wir es erfahren konnten, sind teilweise lokaler Natur, doch überwiegend sind regionäre Rezidive, die endlich den Tod der Kranken herbeiführten.

Fragen wir jetzt, was für Zusammenhang zwischen dem frühzeitigen Melden der Kranken und dem weiteren operativen Erfolg und der definitiven Heilung besteht, so sehen wir, daß es Fälle gibt, bei welchen der Krebs zur Zeit der Operation 1-4 Jahre bestand (5 Fälle) und bei den Kranken keine Rezidive auftraten; hingegen hatten wir auch Kranke, die schon im ersten Jahre die Klinik aufsuchten und dennoch Rezidive auftraten. Hieraus können wir den Schluß ziehen, daß der durch operative Behandlung erzielbare Erfolg zu dem, von den Kranken angegebenen Krankheitsbestand nicht immer proportional ist. Schon die Wahrnehmung des Leidens variiert je nach der Bildungsstufe des Betreffenden, es gibt Leute, die das Vorhandensein einer Warze oder eines Knotens ignorieren und nur das Geschwür als Anfang des Leidens betrachten.

Wenn wir unsere operative Statistik in Prozenten berechnen, so erhalten wir auf die 44 bekannten Fälle bezogen

$$
\begin{aligned}
& \text { rezidivfrei nach } 3 \text { Jahren . . . . 70,45 Proz. } \\
& \text { an Rezidiven gestorben . . . . . 29,55 ", }
\end{aligned}
$$

Thiersch erzielte bei Unterlippenkrebs eine 3 jährige Rezidivfreiheit in 10,4 Proz. seiner Fälle, Bill roth 24,1 Proz., Bergmann 
35,8 Proz., Winiwarter 36,2 Proz., Maiweg 44,5 Proz. und Bruns 66 Proz. Aus Wölflers Klinik gibt Elbel auf Grund von 112 Fällen eine definitive Heilung von 65 Proz. an. Es ist zu bemerken, daß bei der statistischen Berechnung der Wölflerschen Klinik auch diejenigen Fälle mit einem eigenen Prozentsatze bedacht wurden, derer weiterer Verlauf unbekannt war; demnach

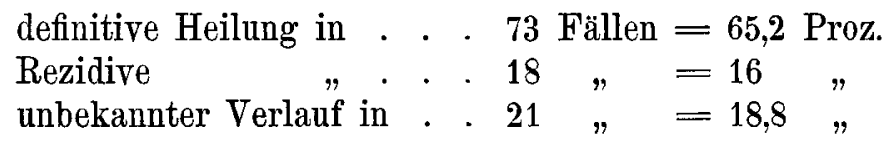

Den günstigen Prozentsatz sah die Klinik nicht davon abhängig, ob sich die Kranken früh in Behandlung begeben, sondern darin, daß sie der Exstirpation des primären Krebses immer die Ausräumung der regionären Drüsen folgen lassen. Auch wir schreiben unsere Erfolge diesem Umstande zu, da wir es nach Obigem nie versäumten, mit dem Dollingerschen Z-Schnitt die regionären Drüsen gründlich zu entfernen.

Nehmen wir die 5jährige Rezidivfreiheit als Basis, so hatten wir 31 Fälle, deren weiterer Verlauf uns in 25 Fällen bekannt ist Von diesen sind

$$
\begin{aligned}
& \text { rezidivfrei nach } 5 \text { Jahren . . } 18 \text { Fälle } \\
& \text { an Rezidiven gestorben . . . } 7 \text {. }
\end{aligned}
$$

3 dieser rezidivfreien Fälle sind $6 \frac{1}{2}, 5$ Fälle 6 Jahre nach der Operation.

Unser Ergebnis in Prozenten ausgedrückt und dasselbe auf die 25 bekannten Fälle bezogen, haben wir

rezidivfrei nach 5 Jahren . . 72 Proz.

an Rezidiven gestorben . . . 28 "

\section{B. Rezidivkrebse.}

Die Zahl unserer Rezidivoperationen wegen Unterlippenkrebs in dem bestimmten Zeitraum beträgt 15. Die erste Operation wurde in 7 Fällen bei uns, in 8 Fällen anderswo vollzogen. Die Zeitdauer zwischen dem Beginne des Krebses und der ersten Operation variierte von 3 Monaten bis zu 12 Jahren in folgender Verteilung: 
der Krebs bestand seit 3 Monaten in . . 1 Falle



Das Auftreten der ersten Rezidive nach der ersten Operation erfolgte nach 15 Tagen bis zu 7 Jahren.

$$
\begin{aligned}
& \text { Rezidiv nach } 15 \text { Tagen in . . . } 1 \text { Falle }
\end{aligned}
$$

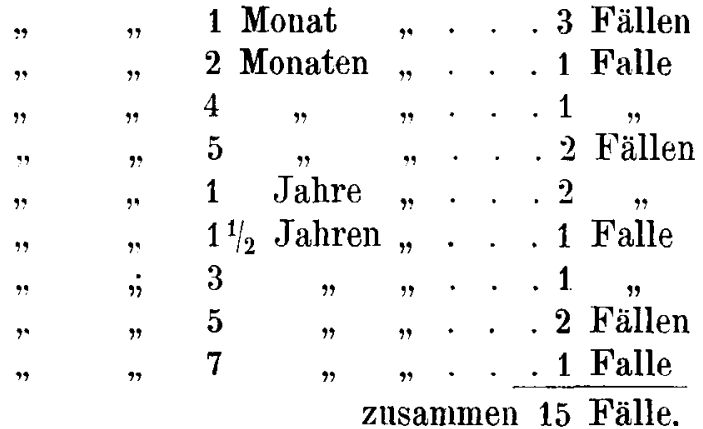

Aus dieser Tabelle sehen wir, daß wir es in vier Fällen mit einem nach 3 Jahren aufgetretenen, sogenannten Spätrezidiv zu tun hatten. Nicht immer waren wir in der Lage, die ersten Rezidive zu operieren, besonders in jenen Fällen nicht, wo schon der erste Eingriff anderwärts vollzogen worden war; in 12 Fällen kamen die Kranken mit I. Rezidive, in 2 Fällen mit II. und in 1 Falle mit IV. Rezidive zu uns. Die Rezidive, mit denen die Kranken uns aufsuchten, waren in 5 Fällen rein lokale, in 5 Fällen lokale mit Metastasen in der Umgebung, in 2 Fällen lokale mit Metastasen in der Umgebung und Erstreckung auf den Unterkiefer, in 2 Fällen lokale mit Metastasen der Umgebung und Erstreckung auf den Unterkiefer und das Zahnfleisch: und endlich in 1 Falle Lokalrezidiv mit Metastasen der Umgebung und Erkrankung des Zahnfleisches und der Wange. 
In diesen 15 Fällen genügte nicht immer eine Operation, um den Kranken ohne Rezidive entlassen zu können, ja wir hatten Fälle darunter, wo Patient trotz mehrmaligen Eingriffs die Klinik mit einem inoperablen Rezidiv verließ. So vollzogen wir:

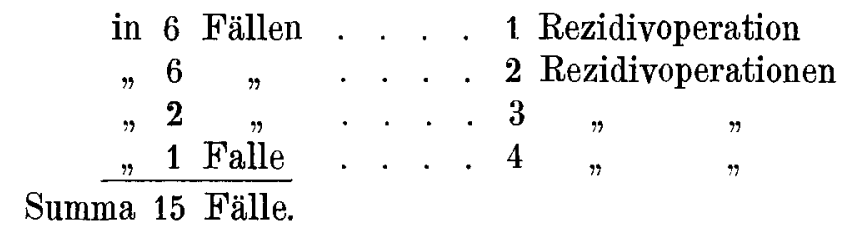

Wir machten daher an 15 Kranken 28 Rezidivoperationen; zählen hier auch noch die ersten Operationen mit, so wurden 15 Kranke 43 mal teils bei uns, teils anderwärts operiert, so daß auf einen Kranken durchschnittlich 3 Operationen fallen. Sehen wir uns nach den ferneren Resultaten unserer Operationen um, so erfuhren wir aus unseren Erkundigungen, daß sich in 3 Fällen nach 5 Jahren keine Rezidive gezeigt hatten. In diesen 3 Fällen bestand der Krebs bei der ersten Operation seit 3 Monaten, bezw. seit 11/4 Jahr (in einem Falle unbekannt), zur Zeit der Rezidivoperation seit 15 Tagen, 3 Monaten und 1 Jahre; alle 3 Fälle waren mit regionärer Drüseninfiltration komplizierte Rezidive. In diesen 3 Fällen genügte ein Eingriff zur Verhütung weiterer Rezidive. Wir erzielten dieses günstige Resultat also in jenen Fällen, wo die Kranken mit erstem Rezidiv in Behandlung kamen, und wir es mit Lokalrezidiven mit geringen regionären Metastasen zu tun hatten. Den Zeitpunkt des Auftretens der Rezidive in jenen 12 Fällen, wo wir kein definitives Resultat erzielen konnten, haben wir schon oben erwähnt; nun wollen wir zeigen, in welchem Zeitraume die nach der letzten Rezidivoperation auftretenden neuen Rezidive den Kranken töteten. Nach:

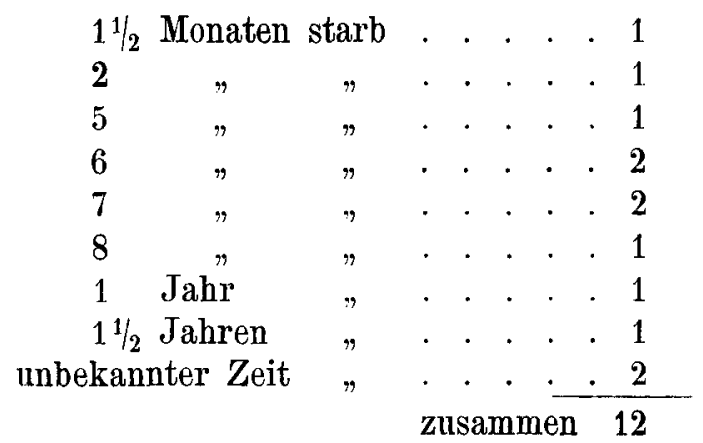


Wenn wir nun die Frage beantworten wollen, wie viel Zeit seit dem angeblichen Beginne des Krebses bis zum Tode bei Rezidivoperationen verstrich, so erhalten wir folgende Übersicht: der Tod trat ein

$$
\begin{aligned}
& \text { nach } 6 \text { Monaten in . . . . } 1 \text { Fall }
\end{aligned}
$$

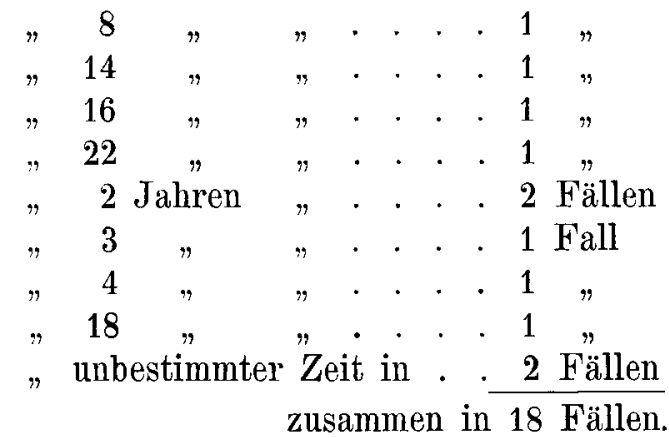

Auf Grund dieser Tabelle können wir unsere Erfahrungen in folgendem zusammenfassen:

1. Die Rezidive waren rein lokale genau in 33,3 Proz. der Fälle, lokale mit regionären Metastasen in 33,3 Proz., auf die Umgebung übergreifend in 33,3 Proz.

2. Die ersten Rezidive traten in mehr als der Hälfte der Fälle innerhalb der ersten 6 Monate ein, in 25 Proz. der Fälle erst nach 3 Jahren als Spätrezidive.

3. Die von uns mit Erfolg operierten Fälle waren sämtlich erste Rezidive.

4. Mehrmalige Operation der Rezidive verlängerte das Leben der Kranken.

Dieselben Erfahrungen machte auch die Wölflersche Klinik. Stellen wir nun unsere Erfolge zusammen, so haben wir von 15 Fällen (deren weiteres Geschick bekannt):

rezidivfrei nach 3 Jahren . . . 5 Fälle $=20$ Proz. an Rezidiven gestorben . . . . $12 "=80$ "

Wölflers Klinik weist ebenfalls nach I. Rezidivoperation, 3 Jahre als Basis genommen, 11,7 Proz. Heilung auf.

Nehmen wir die 5jährige Rezidivfreiheit als Grundlage, so hatten wir in 11 Fällen (wo der weitere Verlauf bekannt):

rezidivfrei nach 5 Jahren . . . . 3 Fälle

an Rezidiven gestorben ... . 8 " 
Bei zweien der rezidivfreien Fälle sind nach der Operation 6 Jahre verflossen. Unserm Ergebnis nach, in Prozenten ausgedrückt, hatten wir:

rezidivfrei nach 5 Jahren . . . . 27,3 Proz.

an Rezidiven gestorben . . . . 72,7 "

\section{Der Krebs der Mund-, Rachenhöhle und der Wange.}

Das Carcinom der Mundhöhle und der Wangen gehört zu den bösartigsten, da die Infiltration der umgebenden Gewebe und Drüsen rasch eintritt. Gussenbau er bemerkte, daß schon in einer kleinen, sich normal anfühlenden Drüse ein Krebsherd sein könne, daher auch jene Drüsen in der Nähe des primären Krebses verdächtig sind, welche noch keinerlei makroskopische Veränderung aufweisen. Da sich aber die Lymphbahnen kreuzen, erkranken nicht nur die auf der Seite des primären Krebses befindlichen Drüsen, sondern auch jene der entgegengesetzten Seite. Dies beobachteten auch wir in einer Reihe unserer Fälle. Die Erklärung ist darin zu suchen, daß die von der Submaxillaris und Submentalis-Drüse ausgehenden Lymphwege, in welche die Lymphgefäße der Wange münden, in die beiderseits gelegenen tiefen Halsdrüsen ergießen, so daß sich hier den Krebszellen Gelegenheit zur Ansiedlung, oder embolischen Verschleppung bietet. Weiterhin wissen wir aus den Forschungen Pólyas und Navratils, daß sich die Lymphbahnen der Wangen und des Zahnfleisches in die submaxillaren, submentalen und Parotis-Drüsen, ferner in die oberflächlichen und tiefen Drüsen des Halses ergießen, und daß diese Bahnen mit den Periost des Unterkiefers in enger Verbindung stehen. Nach Küttner münden die Lymphbahnen des Mundgrundes in die supraclavicularen Drüsen, so daß das Bett der Fortführung der Krebszellen ein sehr weites ist. Demzufolge sehen wir oft, daß ein von der Wangenschleimhant ausgehender Krebs auf das Periost des Unterkiefers übergreift und die beiderseitigen submaxillaren und Halsdrüsen infiltriert sind. Nach Dollingers Erfahrungen sind bei dieser Krebsart häufig auch die tiefen oberen Halsdrüsen mit in Bereich gezogen.

Die anamnestischen Daten ergaben in 14 Fällen, daß Patient entweder starker Raucher ist, oder dem Tabakkanen frönt, und den Kautabak in der später carcinomatös gewordenen Wangenhälfte zu halten pflegte, und so finden wir an den Zähnen häufig 
Schmutz, Stein, auf der Schleimhant Leukoplaques. Interessant ist der Fall bei einem 36jährigen Manne, daß jene Wangenhälfte carcinomatös erkrankte, auf welcher er permanent Tabak kaute; oder der Fall, wo der Kranke gegen Zahnschmerz Ätzmittel anwendete, und der Krebs von der hierdurch hervorgerufenen Ätzwunde ausging.

Daß der Krebs der Wange und der Mundhöhle nicht ausschließlich eine Erkrankung des Greisenalters ist, beweisen ein 24jähriger Mann, bei dem der weiche Gaumen und die Tonsille zu einem hühnereigroßen Tumor geworden waren, und folgende Tabelle:

Es meldeten sich im Alter

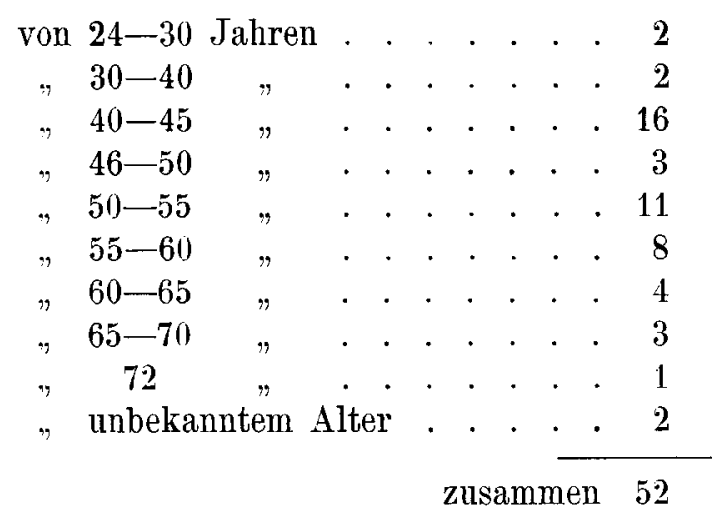

Die Erkrankung fällt demzufolge meistens zwischen dem 40. bis 55. Lebensjahr. Dem Geschlechte nach waren es sämtlich Männer, mit Ausnahme einer Frau, bei der Carcinom des Arcus palato-glossus, der Gingiva und der Wange vorhanden war. Der Ausgangspunkt des Krebses war:

das Zahufleisch in . . . . . . . 9 Fällen

der Gaumen und die Tonsillen in . . 10 "

die Wangenschleimhaut in, . . . 33 "

Unter 33 Wangenkrebsen waren 23 linksseitige. Die Tumoren waren größtenteils exulzeriert, die Geschwüre mit der Umgebung eng verwachsen, von der Größe einer Krone bis Kindeshandfläche. Der Zeitraum des Anfanges der Krankheit bis zur Aufnahme in die Klinik variierte von 3 Wochen bis zu 2 Jahren mit folgender Verteilung: 
Es kamen

$$
\begin{aligned}
& \text { zwischen dem 1.-3. Monat . } 11 \text { Kranke }
\end{aligned}
$$

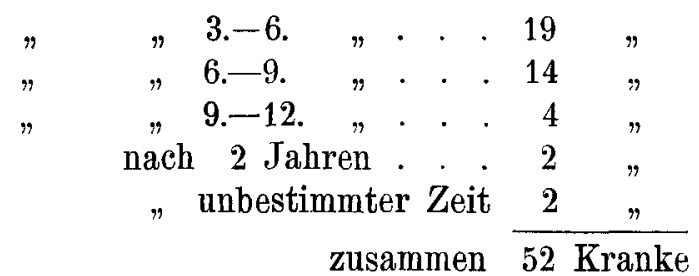

Zur leichteren Übersicht des operativen Erfolges wollen wir diesen Abschnitt in 3 Gruppen behandeln.

a) Krebs des Zahnfleisches.

Bei 4 vom Zahnfleisch ausgehenden Carcinomen operierten wir radikal. In 2 Fällen saß der exulzerierte Tumor am Unterkiefer in der Gegend der beiden Schneidezähne, in 2 anderen am Proc. alveolaris des Oberkiefers. Zuerst entfernten wir die regionären Drüsen mit dem Kocherschen Gurgel- oder dem Dollingerschen Z-Schnitt. Die Tumoren waren von großer Ausdehnung, so daß wir in einem Falle, um gründlich exstirpieren zu können, das Kieferbein nach Sédillot entzwei sägen mußten, in einem anderen Falle waren wir gezwungen, eine partiale Resektion des erkrankten Kiefers vorzunehmen nach vorheriger Unterbindung der Carotis externa, Tracheotomie und Rachentamponade; in einem dritten Falle war auch die Resektion des Ductus stenonianus notwendig.

Operative Mortalität hatten wir in einem Falle zu verzeichnen, als Patient nach der Resektion des Oberkiefers eine Bronchopneumonie bekam. Die Sektion wies übrigens auch auf der Pleura Krebsmetastasen auf.

Rezidiv wurden 2 Fälle nach 1-2 Monaten. Die Rezidive töteten den Kranken nach 3-4 Monaten. Rezidivfrei blieb ein Fall. Dies war ein 57jähriger Mann, welcher bei der Aufnahme in der Gegend der Schneidezähne des Unterkiefers, am Zahnfleische unter dem Zungenfrenulum, einen Tumor von der Größe einer wälschen Nuß hatte. Die Operation bestand im Ausräumen der Unterkiefergegend, und nach Unterbindung der Art. lingualis in der Exstirpation des durch Entzweisägen des Unterkiefers freigelegten Tumors. Patient hat heute, 7 Jahre nach der Operation, wie er uns schreibt, „keine Beschwerden“ und bildet einen jener seltenen Fälle von Rezidivfreiheit, die in der Literatur verzeichnet sind. 
b) Der Wangenkrebs.

Wangenkrebs operierten wir in 17 Fällen radikal. Die Indikation der Operation des Wangencarcinoms geschieht nach Prof. Dollinger innerhalb weiter Grenzen, indem, ohne auf die Ausdehnung des Tumors Rücksicht zu nehmen, die Indikation einer Radikaloperation immer vorliegt, solange auf eine Entfernung aller ergriffenen Teile zu hoffen ist, und der Kranke nicht so herabgekommen ist, daß er den Eingriff voraussichtlich nicht aushalten kann. Die Indikation stellt Prof. Dollinger auf Grund jener Prognose, welche sonst dem Wangenkrebs-Kranken zukommt. Einesteils stellen sich schon in den ersten Stadien der Krankheit Schmerzen ein; später gesellt sich Mundsperre hinzu, welche die Ernährung verhindert und es dem Kranken unmöglich macht, sich von dem Sekret des carcinomatösen Geschwüres zu befreien, so daß er einer Bronchopneumonie anheimfällt; weiterhin breitet sich das Übel auf den Grund der Mundhöhle, die Zunge und den Rachen aus, so daß Schlingbeschwerden entstehen; endlich durchbricht das Geschwür die Hant und führt zu profusen Blutungen. Eben darum muß operiert werden, sollten wir auch nur das Leben des Kranken durch ein schmerzloses Intervall verlängern.

Der Ausgangspunkt des Wangenkrebses war in unseren Fällen meistens die hintere bucco-gingivale Falte vor dem aufsteigenden Teile des Unterkiefers, doch kamen die Kranken nur dann in Behandlung, wenn das Krebsgeschwür schon einen großen Teil der Wangenschleimhaut ergriffen hatte.

Auch Küster und Mikulicz halten die hintere Wangentasche für den häufigsten Ausgangspunkt. In einem unserer Fälle entwickelte sich der Krebs um die Papille des Ductus stenonianus.

Das operative Verfahren besteht auf unserer Klinik darin, daß wir uns nicht nur auf die Entfernung des primären Krebses beschränken, sondern die regionären Drüsen immer entfernen. Die Drüsenexstirpation ging in jenen Fällen, wo ein Decken des entstandenen Defektes nach Entfernung des Tumors durch Halslappen voraussichtlich nicht nötig war, der Operation in der Mundhöhle voraus. In jenen Fällen jedoch, wo wir dem Halse einen Lappen entnehmen mußten, entfernten wir die Drüsen nur nach der Operation des Wangenkrebses.

Eine Regel für die Art und Weise der Entfernung des Wangencarcinoms läßt sich nicht aufstellen, da dies durch den Sitz und die Ausdehnung des Krebses bedingt wird, doch operierten wir 
immer möglichst radikal, so daß sich in 4 Fällen selbst die Resektion des Unterkiefers als nötig erwies, da der carcinomatöse Prozeß weit vorgeschritten war. Auch unser plastisches Verfahren war nicht immer das gleiche, sondern dort, wo uns das Schließen eines Schleimhautdefektes nicht gelang, bedienten wir uns der Gersunyschen, Israelschen und Staffelschen Plastiken.

Unter unseren Fällen waren auch solche, welche schon anderwärts in Behandlung gewesen waren; so wurde z. B. in einem Falle der Thermokauter schon 5 mal angewendet; in einem anderen Falle unterzogen wir einen Kranken, der 2 wöchentlich auftretenden Rezidive wegen, 7 Operationen und es kamen auch solche vor, bei welchen wir wegen auftretenden Rezidiven 2-, 3 mal operieren mußten.

Von 17 Fällen ist uns der weitere Verlauf in 10 Fällen bekannt. In 7 Fällen trat ein meist lokales Rezidiv ein, obwohl es uns gelang zu erfahren, daß in 3 Fällen auch regionäre Metastasen sich einstellten. Das Rezidiv entwickelte sich nach einer Zeit von 2 Wochen bis zu einem Jahre und endete innerhalb von 6-12 Monaten letal. In einem unserer Fälle jedoch trat das Rezidiv nach der Operation eines auf die Wange, das Zahnfleisch des Unter- und Oberkiefers sich ausbreitenden Carcinoms nach 2 Jahren auf, nachdem Patient vorher einen Hufschlag ins Gesicht erlitt. Dies führte nach Angabe der Familienangehörigen zum Rezidiv, welches den Kranken nach 11/2 Jahren tötete.

In 3 Fällen sind nach der Operation $3 \frac{1}{2}-3$ Jahre verflossen und die Kranken sind rezidivfrei; in einem Falle konnten wir uns hiervon persönlich überzeugen, in 2 Fällen schreiben uns die $\mathrm{Pa}$ tienten, daß an ihrem Befinden nichts auszusetzen ist. In diesen Fällen bestand der Tumor seit $3-5$ und 7 Monaten und war bereits in einer Ausdehnung von einer Krone bis zur Größe eines Fünfkronenstückes exulzeriert. In allen 3 Fällen räumten wir vor der Entfernung des primär erkrankten Gewebes die regionären Drüsen aus.

Unser Resultat in Prozenten ausgedrückt und bloß auf die uns bekannten Fälle bezogen, hatten wir

rezidivfrei nach 3 Jahren. . . . 30 Proz. an Rezidiven gestorben. . . . 70 "

Dieses Resultat ist also auch für das fernere Ergebnis der Wangenkrebsoperation zufriedenstellend, besonders wenn wir bedenken, wieweit wir die Grenzen der Wangenkrebsoperation zogen. 
Morestin kann im Jahre 1900 anf dem Kongreß von definitiver Heilung überhaupt nicht sprechen, da seine Patienten binnen 11/2 Jahren sämtlich an Rezidiven gestorben sind.

c) Der Krebs des Gaumens und der Tonsillen.

Gaumen- oder Tonsillenkrebs operierten wir in 3 Fällen. In 2 Fällen entfernten wir das Krebsgeschwür mit dem Wangenquerschnitt, in einem Falle nach der temporären Resektion des aufsteigenden Unterkieferastes nach Langenbeck.

Operative Mortalität hatten wir in einem Falle, als wir wegen präventiver Stillung der Blutung die beiderseitige Art. Carotis ext. unterbanden. Den Tod führte die von der Unterbindungsstelle der Carotis ext. in die Carotis int. sich verbreitende Thrombose und die infolgedessen auftretende Emollitio cerebri herbei. In einem Falle entwickelte sich ein Lokalrezidiv nach 6 Monaten, welches 6 Monate nach dem Auftreten zum Tode des Kranken führte. In einem Falle handelte es sich um ein seit 4 Monaten bestehendes Geschwür auf der linken Tonsille, welches auch auf den Arcus palato-glossus hinübergegriffen hatte. Nach vorhergegangener Ausräumung der regionären Drüsen entfernten wir das Carcinom mittels Wangenquerschnitt; heute, 3 Jahre nach der Operation, ist der Kranke rezidivfrei und befindet sich, wie er uns mitteilt, wohl.

Fassen wir die Krebse der Mundhöhle zusammen, nämlich den Zahnfleischkrebs (Gruppe A), den Wangenkrebs (Gruppe B) und den Krebs des Gaumens und der Tonsillen (Gruppe C), so ersehen wir hieraus, daß wir in 24 Fällen radikal vorgingen; bekannt ist der weitere Verlauf in 17 Fällen, hiervon

operative Mortalität in . . . 2 Fällen = 11,7 Proz.

rezidivfrei nach 3 Jahren . . 5 Fälle $=29,4$ "

Rezidive in . . . . . . 10 Fällen $=58,8$ "

\section{Der Krebs der Zunge und das sublinguale Carcinom.}

Den Krebs der Zunge von dem sublingualen Carcinom zu trennen geht nicht an, da das anf der Zunge sitzende primäre Carcinom den Grund der Mundhöhle oft rasch infiltriert. In unseren Fällen 
war der Grund der Mundhöhle fast immer infiltriert, ob jetzt der Krebs vom Zungenfrenulum, von der Zungenoberfläche oder von der Zungenseite ausging. Nach Sachs ist der Grund der Mundhöhle in 37,9 Proz., nach Meyer in 40 Proz., nach Winiwarter in 45,4 Proz. und nach Wölfler in 57,5 Proz. der Fälle carcinomatös infiltriert, so daß es leicht begreiflich ist, weshalb der Krebs der Zunge zu den bösartigsten dieser Gruppe gehört. Diese Malignität wird mit den allatomischen Verhältnissen der Lymphbahnen der Zunge erklärt. Aus den Untersuchungen Küttners wissen wir, daß die Lymphe einer Zungenhälfte in die beiderseitigen Drüsen gelangt, und daß die Lymphwege der Zunge und der regionären Schleimhaut zu einer und derselben Stelle führen. Abführende Lymphbahnen sind in großer Anzahl vorhanden und zwischen ihnen sind zahlreiche Anastomosen. Die regionären Lymphdrüsen der Zunge sind zahlreich, da die Glandulae cervicales profundae, submaxillares und linguales alle mit den Lymphbahnen der Zunge kommunizieren. Die Hauptlymphdrüse der Zunge sitzt auf der Vena jugularis in der Höhe der Verzweigung der Carotis; zwischen der Zunge und den supraclaviculären Drüsen besteht eine direkte Verbindung. Diesen anatomischen Verhältnissen entsprechend sahen wir bei einem auf die eine Zungenhälfte lokalisierten Krebs die beiderseitigen Drüsen carcinomatös infiltriert und daß bei einem auf der linken Seite der Zungenwurzel sitzenden Krebse die rechtsseitigen Halsdrüsen geschwollen waren.

Mit Zungenkrebs und Krebs der Unterzungengegend gelangten 38 Kranke zur Aufnahme, darunter 2 weiblichen Geschlechts. Daß Zungenkrebs auch in jüngeren Jahren auftreten kann, beweist unsere Statistik, in welcher ein Fall im 26. Lebensjahre verzeichnet ist. Wölfler beobachtete bei einem 17jährigen Kranken Zungenkrebs. Die Verteilung dem Alter nach zeigt folgende Tabelle. Es meldeten sich:

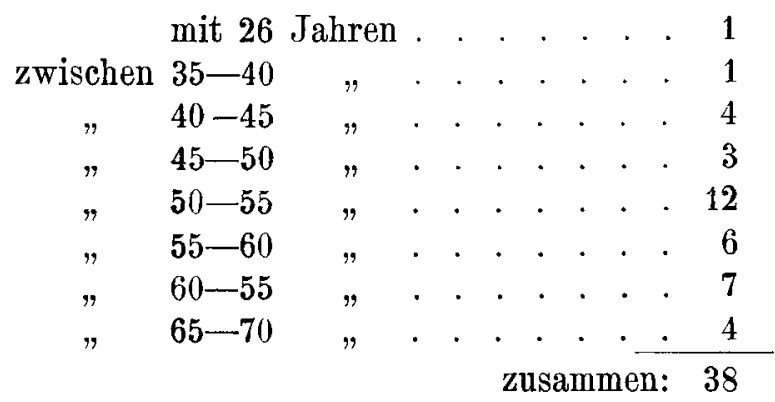


Die Mehrzahl der Fälle fiel also zwischen das 50.--55. Lebensjahr. In den anamnestischen Daten finden wir häufig Abusus in Baccho und starken Nikotingenuß verzeichnet. In vielen Fällen befiel der Krebs pathologisch veränderte Gewebe, so bildete in einem Falle eine seit 6 Jahren bestehende Warze den Ausgangspunkt, in einem anderen Falle entwickelte sich das Carcinom in einer Bißwunde. In einem Falle bildete ein scharfer kariöser Zahn den Ausgangspunkt, welcher die Zunge längere Zeit hindurch reizte. In 2 Fällen giıg Lues vorans und in einem der beiden Fälle entwickelte sich seit 2 Monaten der Krebs in der seit 11/2 Jahren vorhandenen luetischen Narbe. In diesem Falle bestätigte auch die Probeexzision die makroskopische Diagnose. In einem Falle verbreitete sich das Carcinom nicht nur per continuitatem, sondern auch per contactum, da nach einem, auf der rechten Zungenseite sitzenden exulcerierten Krebsgeschwür, auf der gegenübergelegenen Schleimhautpartie der Wange ein carcinomatöses Geschwür auftrat.

Mehrmals beobachteten wir in der Gegend des Krebses Leukoplakie, auf deren ätiologische Wichtigkeit schon Volkmann und Billroth hingewiesen haben, später machten Schuchardt und zuletzt Bozzi auf diese Plaques aufmerksam.

Die in den ersten 4 Jahren vollzogenen 13 Operationen ergeben eine traurige Statistik. Den Grund müssen wir in der Schwere der Fälle suchen, da wir es in keinem Falle mit einem nur auf der Zunge lokalisierten Carcinom zu tun hatten, sondern schon die ganze Zungengegend carcinomatös infiltriert war. Auch der Zungenkrebsoperation ging immer die Ausräumung der regionären Drüsen voran. Die Zungenamputation vollzogen wir entweder mit einfacher Mundspreize oder mit Wangenquerschnitt oder mit der temporären Resektion des Unterkiefers nach Langenbeck oder mit Entzweisägen des Kiefers in der Mitte nach Sédillot. In nenerer Zeit vermeidet Prof. Dollinger womöglich den Wangenquerschnitt und das Entzweisägen des Kieferbeins. Den primären Krebs entfernt er entweder per os oder mittels Pharyngotomia lateralis, da so die Ernährung und Schlingbewegungen des Kranken weniger beeinflußt sind und die Patienten der Gefahr einer Schluckpneunomie weniger ansgesetzt werden.

Operativen Tod hatten wir nach den 13 Operationen in 5 Fällen zu verzeichnen: 2mal Schluckpneumonie, 1 mal Sepsis, 1 mal fettige Myodegeneration des Herzens; in einem Falle arrodierte ein in der Tiefe des Halses auftretender eiternder Prozeß die Carotis ext. und den Tod führte die profuse Blutung herbei. 8 Kranke verließen 
die Klinik geheilt, doch führte innerhalb eines Jahres meistens nach 5 bis 6 Monaten auftretendes Lokal- oder Regionärrezidiv zum Tode.

Die Ergebnisse anderer Operateure sind folgende: Winiwarter beobachtete eine Rezidivfreiheit nach 3 Jahren von 46 Fällen 4 mal, Kocher (1881) von 14 Fällen 4 mal, Krause von 37 Fällen 3 mal. Poirier beobachtete in 5 Fällen eine 33 resp. 22 monatliche Rezidivfreiheit. Binder hatte auf der Züricher Klinik von 33 Fällen innerhalb eines Jahres 16 Rezidive. Von Mikuliczs 19 Fällen starben 14 innerhalb 2 Jahren. Von Czernys 19 Fällen starben 12, bei Krönleins 33 Fällen trat der Tod in 25 Fällen infolge der Rezidive ein. Angesichts dieser schlechten Resultate empfiehlt Wölfler die definitive Heilung bei Zungenkrebs nicht nach 3 Jahren, sondern schon nach einem Jahre auszusprechen, da ja selbst diesen Zeitpunkt sehr wenige erreichen.

\section{Der Kehlkopfkrebs.}

Mit Kehlkopfkrebs lagen 3 Kranke auf der Klinik. In allen Fällen ging der Krebs von den Stimmbändern aus, so daß wir es bisher nur mit einem Carcinoma intrinsicum zu tun hatten, d. h. mit einem in dem Larynx lokalisierten Krebs, welcher häufiger vorkommt, wie außerhalb desselben auf der Epiglottis oder in Sinus pyriformis. Zweimal ging der Krebs von den linksseitigen Stimmbändern aus, einmal von den rechtsseitigen. Das Alter der Kranken war 51, 55 und 61 Jahre, dem Geschlechte nach sämtlich Männer. In derA namnese finden wir einmal starken Tabakgenuß verzeichnet, demzufolge seit Jahren ein Kehlkopfkatarrh bestand; in einem Falle hören wir von einer seit 4 Monaten bestehenden Laryngitis.

Die Ausdehnung des Krebses war in 2 Fällen nicht groß. In dem einen Falle ging der Krebs vom rechten wahren Stimmbande aus und griff etwas auf die linke Kehlkopfhälfte und die Cartilago cricoidea über. Infiltrierte Drüsen sind am Halse nicht fühlbar gewesen. Die endolaryngeale Probeexzision bestätigte die Krebsdiagnose. In diesem Falle vollzog Prof. Dollinger am 31. Oktbr. 1900 die totale Kehlkopfexstirpation. Die Operation wurde in Narkose ohne vorhergegangene Tracheotomie vollzogen. Pat. befand sich post operationem wohl und verließ die Klinik mit dem von Bruns verbesserten künstlichen Kehlkopf. Prof. Dollinger demonstrierte den Kranken auch im Königl. Ärzteverein, und Pat. 
befindet sich heute, 5 Jahre nach der Operation, wohl, und kann, wie er uns schreibt, ,gut essen und trinken."

In einem anderen Falle war der vom linken Stimmbande ausgehende Krebs schon von großer Ausdehnung, griff auf die beiden oberen Knorpelringe der Trachea über, so daß der ganze Kehlkopf mit dem Tumor ausgefüllt war. Die Geschwulst erwies sich als ein Epithelcarcinom. In diesem Falle starb der Kranke am dritten Tage nach der totalen Kehlkopfexstirpation an Sepsis. Im dritten Falle saß der Krebs auf dem linken wahren Stimmbande, und vollzog hier Prof. Dollinger eine partielle Kehlkopfexstirpation; diesen Patienten demonstrierte er am 22. Januar 1901 dem Königl. Ärzteverein. Bei 3 operierten Kranken hatten wir

operativen Tod in . . . . . . . . . 1 Falle

rezidivfrei nach 5 Jahren in . . . . 1 "

geheilt entlassen, weiterer Verlauf unbekannt in 1 Falle.

\section{Der Krebs der Brustdrüse.}

Unter den Tumoren der Brustdrüse nimmt der Krebs die erste Stelle ein. Von September, 1897. bis September 1903 gelangten bei uns 122 Kranke mit der Diagnose eines Brustdrüsentumors zur Aufnahme, wovon sich 108 Fälle als Carcinom erwiesen, was 88,6 Proz. aller Brustdrüsengeschwülste entspricht. Während derselben Zeit meldeten sich 340 Kranke mit Brustdrüsengeschwülsten in Ambulatorium, hiervon 305 Brustdrüsencarcinome, also 83,8 Proz. aller Fälle. In Billroths Statistik erscheint der Krebs in 82 Proz. aller Brustdrüsengeschwïlste, bei Schmidt(Heidelberg) mit 82 Proz., bei Bryant mit 83,16 Proz. und bei Angerer mit 85,5 Proz.

In 108 Fällen saß der Krebs 51 mal in der rechten, 57 mal in der linken Brustdrüse. Das Alter, in welchem der Krebs auftrat, zeigt folgende Tabelle:

$$
\begin{aligned}
& \text { im 23,-26. Lebensjahre . . . . } 2 \text { Fälle }
\end{aligned}
$$

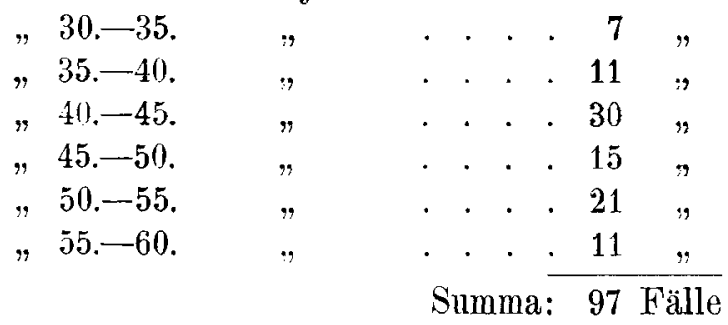




$$
\begin{aligned}
& \text { Übertrag: } 97 \text { Fälle }
\end{aligned}
$$

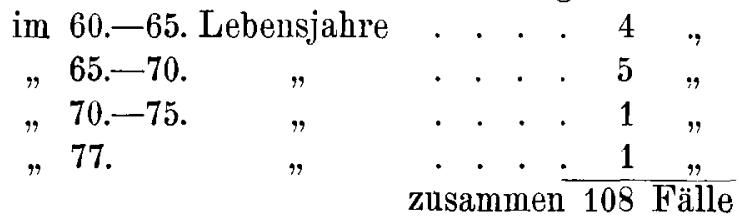

Unsere jüngste Kranke war demnach 23, die älteste 77 Jahre alt. Zwischen dem 40.-55. Lebensjahre waren 66 Kranke, also 56,7 Proz. aller Fälle.

In den anamnestischen Daten spielt das Trauma, welches die Kranken mit Vorliebe erwähnen, $11 \mathrm{mal}$ eine Rolle. Mastitis ging in 2 Fällen voraus vor 2 resp. 3 Jahren, der Verlauf war ein schwerer gewesen, mit langwieriger Eiterung und erforderte mehrmals einen Eingriff. Paget disease beobachteten wir vor Entwickelung des Krebses bei einem unserer letzten Fälle. Heredität finden wir nicht erwähnt.

Auf unserer Klinik waren 2 Operationsmethoden gebräuchlich. In den Jahren 1597/8 und 1898/9 war unser Verfahren folgendes

1. Entfernung der ganzen Brustdrüse,

2. Entfernung der Faszie des großen Pektoralis samt den oberflächlichen Schichten des Muskels,

3. Exstirpation der Drüsen der Achselhöhle samt dem Fettge webe.

Seit Herbst 1899 operierten wir nach einem vollkommeren Verfahren, da wir die Halsted-Kochersche Methode anwandten. Der Gang der Operation ist folgender:

1. Bloßlegung der Achselgrube und Ausräumung des Fettgewebes samt den Drüsen;

2. Entfernung der erkrankten Brustdrüse samt den beiden Pektorales;

3. sind Drüsen palpabel, die Ausräumung der Fossa supraclavicularis und das vom $\mathbf{M}$. scalenus ant. und M. sternocleidomastoideus gebildeten Dreieck.

Zur richtigen Beurteilung der Operationsresultate wollen wir die nach den verschiedenen Methoden operierten Fälle getrennt behandeln.

\section{Die nach der älteren Methode behandelten Fälle.}

A. Primäre Krebse.

Die Zahl der hierher gehörigen Fälle beträgt 38, von denen der weitere Verlauf bei 25 bekannt ist. Die Zeitdauer zwischen 
Auftreten des Krebses und der Aufnahme auf der Klinik variierte zwischen 2 Monaten und 4 Jahren. Die Mehrzahl der Fälle kam wach dem ersten Jahre in einem Zeitpunkte, wo der Krebs nicht mehr lokalisiert war, sondern den M. pectoralis major schon infiltriert hatte. In diesen Fällen konnte also jene Methode, welche bloß die Faszie und die oberflächlichen Schichten entfernt, schon a priori nicht genügen, da durch die zurückgelassenen Krebskeine das Rezidiv sich rasch einstellt.

Detaillieren wir in den 25 bekannten Fällen die zwischen Auftreten des Übels und Beginn der Behandlung verstrichene Zeit, so erhalten wir folgende Tabelle. Es meldeten sich:

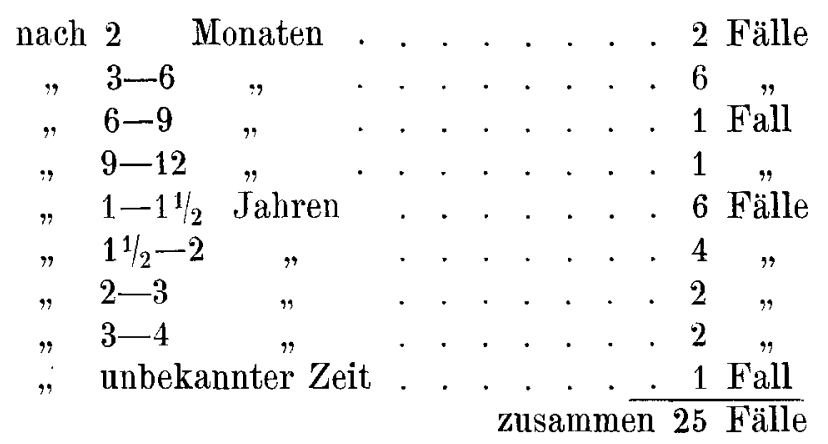

In 10 Fällen, d. h. bei 40 Proz. fanden wir Exulzeration vor; der Krebs war von Hühnerei- bis Mannesfaust- ja sogar von Kindskopfgröße und mit der Umgebung eng verwachsen.

Operative Mortalität hatten wir in 2 Fällen zu verzeichnen; in einem Falle trat nach der Operation eines exulzerierten Krebses Sepsis auf, in einem anderen führte nach 7 Tagen eine Pleuritis cancrosa zum Tode.

An Rezidive, starben 17 Kranke; die Rezidive traten nach 3 Monaten bis $2 \frac{1}{2}$ Jahren auf und waren entweder lokal oder regionär. Die Rezidive traten auf:

$$
\begin{aligned}
& \text { nach } 3 \text { Monaten in . . . . . . } 2 \text { Fällen }
\end{aligned}
$$

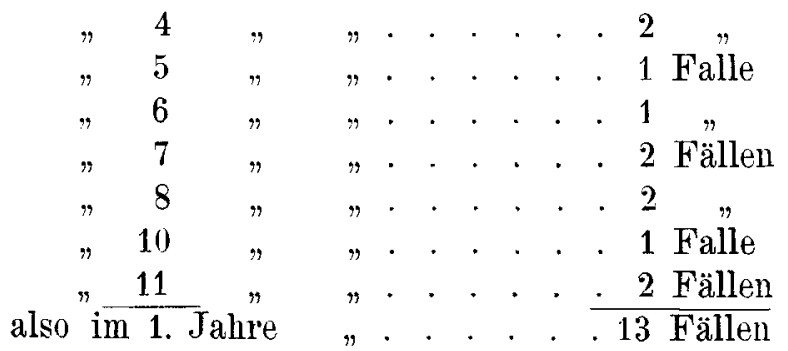




$$
\begin{aligned}
& \text { nach } 14 \text { Monaten in . . . . . } 1 \text { Falle } \\
& \text { " } \quad 218 \text { Jahren " . . . . . . . . . . } 2 \text { Fällen }
\end{aligned}
$$

Die Rezidive führten den Tod im Durchschnitte nach 6 Monaten herbei.

Interkurrente Krankheiten rafiten unseren Erkundigungen gemäß 2 Kranke hin. Bei beiden wurde betont, daß keine Lokalrezidive vorhanden waren.

Rezidivfrei blieben 4 Fälle. Interessant ist hier ein Fall, wo wir bei der 45 jährigen Patientin die Brustdrüse nach dieser Methode amputierten und die Achselhöhle ansräumten. Dies geschah am 16. Februar 1898. Patientin war bis zum Herbste 1904 vollkommen gesund, also $61 / 2$ Jahre lang, und jetzt trat das Rezidiv in der Narbe und eine Metastase in der anderen Brustdrüse auf. Im März 1905 vollzogen wir an der Kranken die Halsted-Kochersche Operation und Patientin lebt heute d. h. im Oktober 1905. In den anderen 3 Fällen bestand der Tumor seit 8 Wochen, 3 Monaten, resp. seit 1 Jahre; In einem Falle waren keine Drüsen fühlbar und auch in den beiden anderen Fällen bloß 1-2 Drüsen der Achselhöhle. Bei diesen Fällen sind in einem Falle 6, in dem anderen 5 Jahre verflossen und die ärztliche Untersuchung bestätigte, daß kein Rezidiv aufgetreten war.

Der Erfolg der älteren Methode war also folgender:

operative Mortalität in . . . . . . 2 Fällen

Tod an Rezidiven in . . . . . . . 17 "

Tod an interkurrenten Krankheiten in 2 " rezidivfrei nach 3 Jahren . . . . . 4 Fälle

Das Verhältnis in Prozenten ausgedrückt Operative Mortalität. . . . . . . . 8 Proz. an Rezidiven gestorben . . . . . 68 " an interkurrenten Krankheiten gestorben 8 " Rezidivfrei nach 3 Jahren . . . . 16 "

Nehmen wir den 5 jährigen Zyklus als Basis, so stehen uns bloß 34 Fälle zu Gebote, wovon 25 bekannt sind. Von diesen sind an Rezidiven gestorben. . . . . 17 Fälle operativer Tod . . . . . . . . 2 " interkurrente Mortalität . , . 2 " rezidivfrei nach 5 Jahren . . . . 2 "

In Prozenten bekommen wir dasselbe Verhältnis wie bei der 3 jährigen Rechnung, da die nach der alten Methode operierten 
Fälle eben in jenen Zeitraum fielen, wo nach der Operation 5 Jahre verflossen sind. Hier ist jedoch eigens zu betonen, daß bei einem der 4 Fälle, welche die 5 jährige Rezidivfreiheit erreichten, sich nach $61 / 2$ Jahren ein Spätrezidiv einstellte.

\section{B. Rezidivkrebse.}

Rezidivkrebse operierten wir in 9 Fällen nach der älteren Methode. Die Rezidive traten in 8 Fällen nach anderswo, in einem Falle nach der bei uns ausgeführten Operation auf.

Das weitere Schicksal ist uns bei zwei Kranken bekannt. In dem einen hatten wir es bloß mit einem lokalen, in dem anderen mit einem lokalen und regionären Rezidiv zu tun. Im ersten Falle waren seit der Operation 6 Monate, im zweiten bereits 1 Jahr verflossen. Zur Zeit der ersten Operation bestand der Krebs seit 6 Monaten, resp. seit 1 1/2 Jahren. Beide Fälle gehören zu den definitiv geheilten, da seit der Rezidivoperation 5, resp. 6 Jahre vergangen sind.

\section{Nach Halsted-Kocher operierte Fälle.}

A. Primäre Krebse.

Im 3jährigen Zyklus kommen 22 Fälle in Betracht, wo das weitere Schicksal uns bei 17 bekannt ist.

Operativen Tod hatten wir in einem Falle an Bronchopneumonie. An Rezidiv starben 7 Kranke, teils war dies lokal, teils regionär. Das Rezidiv trat nach 2, 6, 8 und 10 Monaten, resp. nach $1 \frac{1 / 2}{2}, 1 \frac{1 / 2}{2}$ und 2 Jahren auf. Der Tod erfolgte 3-6 Mon. nach dem Auftreten. Der Krebs bestand zur Zeit der ersten Operation seit:

$$
\begin{aligned}
& 5 \text { Monaten in . . . . . . . } 1 \text { Falle }
\end{aligned}
$$



$$
\begin{aligned}
& 9 " \quad " \quad . \quad . \quad . \quad . \quad . \quad . \quad .1 \% \\
& 1 \text { Jahre ". . . . . . . } 2 \text { Fällen } \\
& 2 \text { Jahren ". . . . . . . } 1 \text { Falle } \\
& 4 \text { " } \\
& \text { zusammen } \frac{1}{7 \text { Fälle. }}
\end{aligned}
$$

All Metastase, ohne lokales Rezidiv starben 2 Kranke. Bei einer trat die Metastase nach $1 \frac{1}{2}$ Jahren in den Knochen, bei der 
anderen nach 3 Monaten im Magen auf und führte in beiden Fällen in kurzer Zeit zum Tode.

Nach 3 Jahren sind 7 Kranke rezidivfrei. In diesen Fällen bestand der Krebs seit $3 \frac{1}{2}$ Monaten bis zu 3 Jahren in folgender Verteilung seit:

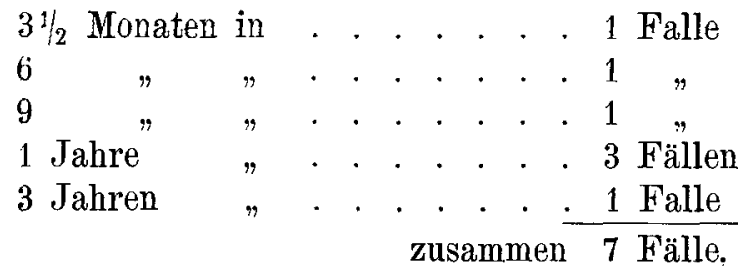

Wir hatten öfters persönlich Gelegenheit, uns von dieser Rezidivfreiheit zu überzeugen. In einem Falle nahm Prof. Dollinger folgenden Status auf: an der Stelle der Operationswunde eine weiße, weiche, verschiebbare Narbe; der Arm bewegt sich sowohl aktiv, wie passiv in jeder Richtung frei. Infiltrierte Drüsen sind nirgends palpabel. Der Kräftezustand ist durchaus befriedigend. Bei einem anderen Falle hob die Kranke in ihrer Antwort direkt hervor, daß sie den Arm nach jeder Richtung frei und gut gebrauchen kann. Diese Fälle beweisen ebenfalls jene klinische Erfahrung, daß die Halsted-Kochersche Operation keine bedeutenderen Funktionsstörungen des Armes nach sich zieht.

Drücken wir unser Resultat in Prozenten aus, und bringen die operative Mortalität in Abzug, so hatten wir bloß die bekannten Fälle gerechnet:

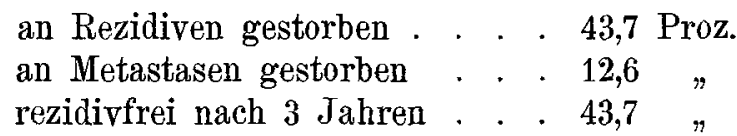

Nehmen wir die in diesem Zeitraume von Prof. Dollinger persönlich operierten 6 Fälle, so haben wir darunter 3 rezidivfrei, also 50 Proz. Den 5jährigen Zyklus können wir nicht berechnen, da seit den Operationen noch keine 5 Jahre verstrichen sind.

Die Statistiken überblickend, sehen wir, daß Billroth, der Winiwarterschen Statistik gemäß, von einer länger als 3 Jahre dauernden Rezidivfreiheit nur in 4,7 Proz. der Fälle sprechen kann. Die definitive Heilung mehrerer Operateure beträgt: Esmarch 11,7 Proz., Hacker 14,51 Proz., Gussenbauer 16,7 Proz., v. Eiselsberg 18 Proz., Ehrich (Klinik zu Rostock, Trendelenburg, Madelung, Garré), 21-21,5 Proz., Czerny (1897) 21 Proz., Küster 21,5 Proz., 
Warren 26 Proz., Helferich 28,5 Proz., Bergmann 28,79 Proz., Braun (Göttingen) 28,32 Proz., Mayo Robson 32,2 Proz., Steinthal 33,3 Proz. Halsted erreichte nach der von ihm inaugurierten Methode in 3jährigem Zyklus eine definitive Heilung in 40,1 Proz. Diese Operationen vollzog er meist selbst. Wir sehen also, daß unsere Erfolge denen anderer nicht nachstehen.

\section{B. Rezidivkrebse.}

Bei Rezidivkrebs wandten wir die Halsted-Kochersche Methode nur in einem Falle an. Dieser Fall bezieht sich auf eine 48jährige Frau, deren primärer Krebs anderwärts schon zweimal operiert wurde. Der Krebs war seit der 2. Operation bis zur Größe einer Kindesfaust gewachsen und exulzeriert. Wir operierten den seit 10 Monaten bestehenden Tumor nach der älteren Methode. Das Rezidiv zeigte sich nach 10 Monaten, sowohl lokal, als regionär. Jetzt wandten wir die Halsted-Kochersche Methode an, und Patientin befindet sich heute nach 4 Jahren wohl und blieb rezidivfrei.

\section{Der Magenkrebs.}

Mit Magenkrebs gelangten 59 Kranke zur Aufnahme. Dem. Alter nach:

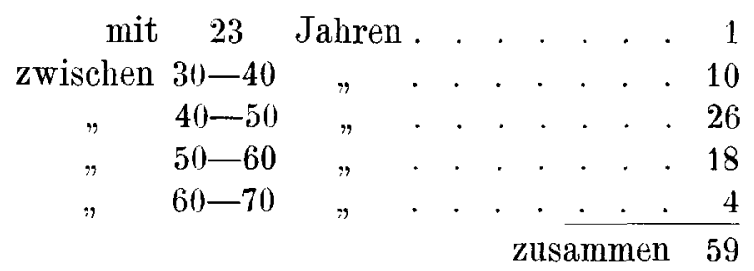

Zwischen 41-60 Jahren entfielen 73,3 Proz. Dem Geschlechte nach waren es 33 Männer und 26 Frauen. Was Vererbung anbelangt, hörten wir in einem Falle erwähnen, daß auch die Mutter an Magenkrebs gestorben ist.

In den anamnestischen Daten finden wir seit längerer Zeit bestehende Magenbeschwerden $47 \mathrm{mal}$ erwähnt. So hatten wir einen Patienten, der seit 22 Jahren über heftige Magenschmerzen klagt und einen, der die Magenbeschwerden an sich seit 10-12 Jahren beobachtet. In einigen Fällen sollen die Kranken vor Entwicklung des Leidens längere Zeit an blutigem Erbrechen gelitten haben, wo sich der Krebs also wahrscheinlich in einer Ulcusnarbe ent- 
wickelte; in einem Falle bestätigte auch die Sektion unsere Annahme, daß der im Antrum pyloricum sitzende Krebs aus einem Ulcus entstand. Der Krebs saß in den operierten Fällen:

an der kleinen Kurvatur in . . . . 2 Fällen

$"$ "großen $"$. . . . 4 "

diffuse an der ganzen Magenwand in 7 "

am Pylorus in . . . . . . . . . 19 "

Die Zahlen beziehen sich bloß auf jene Fälle, wo wir uns vom Ausgangspunkte des Krebses durch die Operation überzeugen konnten. Wir sehen, daß wir es in 59,4 Proz. mit dem Carcinom des Pylorus zu tun hatten.

Die Kranken suchten uns seit dem Bestehen der Krankheit nach verschiedener Zeit auf. Die folgende Zusammenstellung, welche die früher erwähnten Magenbeschwerden außer acht läßt, beweist, wie spät die Kranken den Chirurgen aufsuchen:

Es meldeten sich:



Also 30 Kranke während des ersten Jahres und 29 nach dem ersten Jahre; im Durchschnitte suchen also die Kranken den Chirurgen im 8. Monate auf. Von Krönlein wissen wir, daß der Magenkrebs gewöhnlich nach einem Jahre den Kranken tötet. Wenn wir nun bedenken, daß die Patienten den Chirurgen im 8. Monate 
vom Bestehen des Übels aufsuchen, also nach Ablauf von $2_{3}$ der Zeit, welche die Krankheit im Durchschnitt währt, können wir leicht einsehen, daß wir bloß in einem geringen Prozentsatz der Fälle radikal vorgehen können (siehe Punkt C). Der pathologische Grund liegt darin, daß sich der Krebs ungemein rasch verbreitet und die umliegenden Gebiete in kurzer Zeit mit in den Prozeß einbezieht. In welchem Maße dies bei unseren Fällen geschah, ersehen wir aus folgender Tabelle.

Es waren in den operierten Fällen bereits infiltriert:

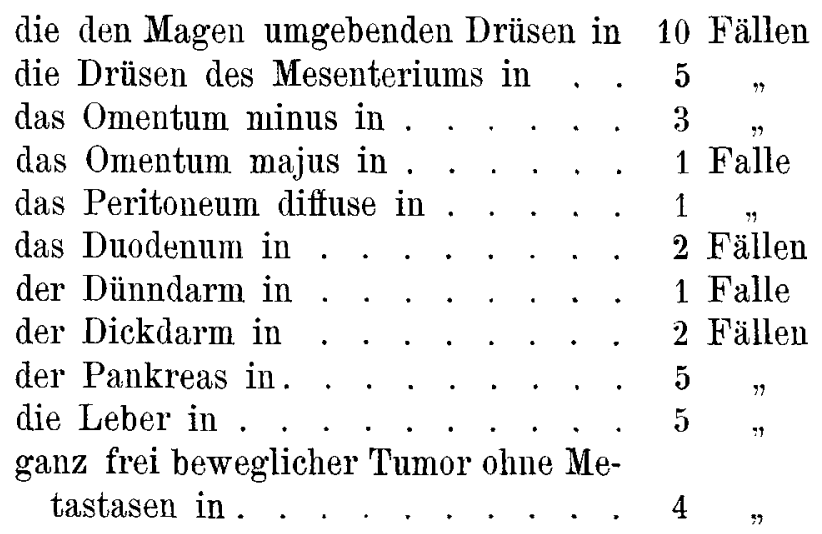

Betrachten wir nach dieser Einleitung einzeln unsere operativen Eingriffe. Diese können wir in 3 Gruppen einteilen:

\section{A. Probelaparotomie.}

Unter 19 Fällen nahmen wir in 4 bloß die Probelaparotomie vor, da diese Fälle zu radikalem Vorgehen nicht geeignet waren; auch ein palliatives Verfahren war nicht am Platze, da sich weder motorische Störungen, noch mechanische Insuffizienz zeigten. Rechnen wir zu diesen 4 Fällen auch jene 8 hinzu, in welchen die Kachexie der Patienten jeden Eingriff kontraindizierte, so sehen wir, daß 30 Proz. unserer Kranken schon inoperabel waren, als sie die Klinik aufsuchten. Auch Krönlein vollzog bei 264 Fällen $73 \mathrm{mal}$ blol die Probelaparotomie und 53 andere Fälle waren schon a priori inoperabel. Von unseren Probelaparotomien starb ein Kranker auf der Klinik, 3 verließen die Klinik mit geheilter Bauchwunde und starben zu Hause innerhalb 30-130 Tagen (im Durchschnitt nach 73) an allgemeiner Erschöpfung. 


\section{B. Palliativoperationen.}

Als Palliativoperation gebraucht Prof. Dollinger zwei Verfahren, nämlich: 1. die Gastroenterostomie; 2. die Jejunostomie.

\section{a) Gastroenterostomie.}

Prof. Dollinger übt die Gastroenterostomia antecolica antica mit Naht aus jenen Gründen, welche er im November 1902 im kgl. ungar. Ärzteverein auseinandersetzte, weil nämlich der Murphysche Knopf häufig in den Magen zurïckfällt, anderseits aber die durch denselben hervorgerufene Öffnung während des Heilungsund Vernarbungsprozesses noch zusammenschrumpft. Auch Mikulicz ist ein großer Anhänger der Naht und sieht die Nachteile des Murphyschen Knopfes in folgendem: 1. da er relativ zu groß ist, fällt er in $1 / 3$ der Fälle in den Magen zurück, 2. ist die durch den Knopf gemachte Öffnung zu klein, 3. kann der Knopf infolge Drucknekrose zu Peritonitis führen, 4. kann er sich nach längerem Gebrauche verstopfen. Unter 8 gastroenterostomierten Fällen starben 4 an Peritonitis, obwohl die Sektion bewies, das die applizierte Naht luft- und wasserdicht geschlossen hat, so daß wir annehmen müssen, daß das septische Sekret des Geschwüres, welches während und nach der Operation in unmittelbarer Berührung mit der Magenund der Darmwunde gelangte, die foudroyante Sepsis hervorrief.

In einem Falle starb der Kranke 8 Tage nach der Operation an Bronchopneumonie, so daß wir unter 8 gastroenterostomierten Fällen 5 operative Todesfälle zu verzeichnen haben. Mit geheilter Banchwunde verließen die Klinik 3 Patienten, welche zu Hause nach 3-6 und 11 Monaten, also durchschnittlich nach $6 \frac{1}{2}$ Monaten starben. Dieses durchschnittliche Alter erreichten auch die Kranken Krönleins.

Später besserte sich die bei Magenkrebs vorgenommene Gastroenterostomie-Statistik, da wir die Kranken auf die Operation gründlicher vorbereiteten und 4-5 Tage hindurch vor der Operation am Vorabend und am Operationstage energische Magenspülungen vornahmen. Die Magenspülungen nehmen wir nicht nur in horizontaler Lage vor, sondern unmittelbar vor der Operation entleeren wir den Magen in "steiler Beckenhochlagerung", die Sonde ziehen wir nur "langsam" heraus, damit auch der letzte Rest des Mageninhaltes herausfließen kann. Durch diese Methode, welche Neck zuerst empfohlen hatte, gelingt es uns, eine völlige Magen- 
entleerung zu erzielen. Seit den oben erwähnten 8 Fällen nahmen wir die Operation in anderen 8 Fällen vor und der Tod trat nur einmal infolge von Peritonitis ein, in 2 anderen Fällen starben die Kranken nicht an Lokalkomplikationen. Einen Fall verloren wir 2) Stunden nach der Operation infolge eingetretener Herzschwäche, den anderen 17 Tage nach der Operation an einer diphtherischen Darmentzündung. Von unseren neuerdings Gastroenterostomierten starben also 12,5 Proz. an lokalen Komplikationen und 25 Proz. an anderen mit der Operation im Zusammenhange stehenden Krankheiten. Die durch andere beobachteten operativen Todesfälle zeigen folgende Zahlen: Ringel 62,2 Proz., Billroth 50 Proz., Carle und Fantino 40 Proz., Kocher 36,3 Proz., Kappeler 32,2 Proz., Mikulicz 31,5 Proz., v. Eiselsberg 31 Proz., Roux 28,1 Proz., und Krönlein 24,3 Proz.

\section{b) Jejunostomie.}

In jenen Fällen, welche für Gastroenterostomie nicht geeignet sind, vollzieht Prof. Dollinger die Jejunostomie und zwar neuerdings die seitliche. Maydlsche Jejunostomie machten wir in dieser Zeit in 3 Fällen. Einen Fall verloren wir an Peritonitis, 2 Kranke aber verließen mit zunehmender Kraft und Gewicht die Klinik und starben nach 1 resp. 4 Monaten, doch wurden die Schmerzen, wegen welcher die Kranken die Klinik aufsuchten, bis zum Tode gelindert.

\section{c) Radikaloperationen.}

Radikaloperationen nahmen wir in 4 Fällen, d. $h$. in 21 Proz. aller Fälle vor. Das Radikalverfahren bestand 2 mal in Pylorus, $1 \mathrm{mal}$ in partieller und einmal in totaler Magenexstirpation. Operativen Tod hatten wir in 2 Fällen. Der eine starb nach einer Pylorusresektion an Peritonitis, der andere nach einer partiellen Magenexstirpation an Schlnckpneumonie. Ein anderer Fall von Pylorusresektion verließ die Klinik geheilt, starb jedoch zu Hause an einem nach 7 Monaten aufgetretenen Rezidiv. Der Kranke, dem Prof. Dollinger am 11. Februar 19(01 den ganzen Magen entfernte, lebt heute 5 Jahre nach der Operation und ist vollkommen gesund und versieht seinen Dienst als Polizist. ${ }^{1}$ )

1) Im erwähnten Zeitraume machten wir nur diese eine totale Magenexstirpation, doch vollzog Prof. Dollinger dieselbe seitdem in 4 Fällen, also insgesamt in 5 Fällen, wovon 2 operative Todesfälle zu verzeichnen sind. 
Die operativen Todesfälle betrugen dei Brüning (Gießener Klinik, 32 Fälle) 18,8 Proz., bei v. Eiselsberg 21 Proz., bei Matti (Kochersche Klinik) 25,7 Proz. und nach Kocherschem Verfahren 16 Proz., bei Czerny 38,5 Proz., bei Mikulicz 44 Proz.

Krönlein berichtet nach 50 Gastrektomien über 14 operative Todesfälle, von den geheilten 36 starben 22 nach $5 \frac{1}{2}$ Monaten bis zu 1 Jahre an Rezidiven, 2 Patienten wurden durch interkurrente Krankheiten dahingerafft; 12 Kranke leben derzeit, wovon bei 10 das Triennium nach der Operation noch nicht verflossen ist, bei 2 hingegen sind bereits 8 resp. 4 Jahre vergangen.

Aus der v. Eiselsberg schen Klinik referiert Clairmont über 33 radikal operierte Fälle (Pylorektomien;) die Operation überstanden 26 Kranke und von diesen leben derzeit 8. 5 Fälle haben das Triennium bereits erreicht; 1 Kranker lebt 7, einer 5, einer 4 und 2 sind schon 3 Jahre nach der Operation.

Aus der Kocherschen Klinik berichtet Matti über 101 radikale Magenkrebsoperationen (Pylorektomien), welche neuerdings ausgeführt wurden. Hiervon leben heute 20 Kranke, also 28,2 Proz. In 12 Fällen sind noch keine 3 Jahre verflossen, bei 8 Fällen jedoch 3, 5 (3 Fälle). 6, 7, 11 und 16 Jahre, was eine definitive Heilung in 9,18 Proz. aller Fälle ergibt.

Auch Czernys Kranker lebt seit 7 Jahren, der Patient Wölflers seit 51/2, der von Maydl seit $8 \frac{1}{2}$, der von Roux seit $9 \frac{1}{2}$ Jahren ohne Rezidiv. Daran schließen sich die beiden von Prof. Dollinger operierten Fälle an, von denen der eine 10, der andere 5 Jahre rezidivfrei ist.

\section{Der Krebs des Dünn- und Dickdarmes.}

Es gelangten 28 Fälle mit Diagnose des Darmkrebses zur Aufnahme: 3 mal war es Dünndarm- und 25 mal war es Dickdarmkrebs.

Um über die Heilerfolge einen genauen Überblick zu erlangen wollen wir die an verschiedenen Stellen des Darmes sitzenden Krebse in eigenen Abschnitten behandeln.

a) Dünndarmkrebs.

In dem erwähnten Zeitraum lagen 3 Kranke mit Dünndarmkrebs auf der Klinik, von denen wir einen operierten. Es war dies ein 56jähriger Mann, der den in seiner Bauchhöhle sich bildenden Tumor vor 6 Wochen bemerkte. Die Operation entfernte das im Intest. jejunum sitzende Carcinom, welches sich als Carcinoma gela- 
tiniforme erwies. Patient verließ die Klinik geheilt, doch ist sein weiteres Schicksal uns unbekannt.

b) Dickdarmkrebs.

Der Sitz des Dickdarmkrebses war in den 25 Fällen:

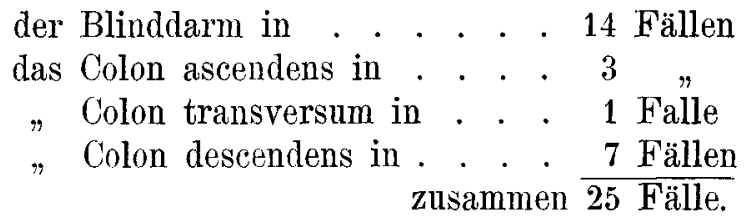

Von den 7 Carcinomen des Colon descendens saßen: in der Curvatura lienalis 1; in der Flexura sigmoidea 3; anderwärts am Colon 3. In 13 Fällen hatten wir mit Carcinoma gelatiniforme zu tun. Dem Geschlechte nach waren es in 21 Fällen Männer und in 7 Fällen Frauen. Das Alter der Kranken betrug:

$$
\begin{aligned}
& 22 \text { Jahre in . . . . } 1 \text { Falle } \\
& 25 \text { " . . . . . } 1 \text {, } \\
& 27 . " . . . .9 .1 \% \\
& \text { zwischen } 30-40, " . . . .2 \text { Fällen }
\end{aligned}
$$

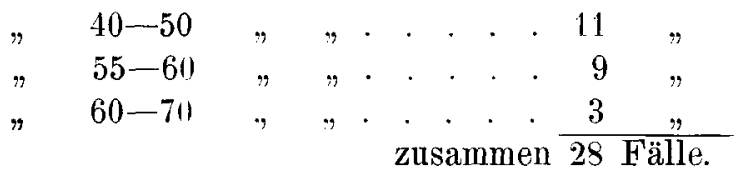

In der Anamnese finden wir das Trauma in 2 Fällen erwähnt. In einem Falle erlitt der Patient vor 5 Monaten durch eine Wagendeichsel einen Stoß in die Bauchgegend, in dem anderen fiel der Kranke vor 5 Jahren von einem Wagen und schlug sich die rechte Banchhälfte stark an; seitdem klagt er über andauernde Kolikschmerzen.

$$
\text { a) Blinddarmkrebs. }
$$

Bei Blinddarmkrebs operierten wir in 5 Fällen. Palliativ operierten wir 3 mal. Einmal erweiterten wir bloß die Fistel, eines mit der Bauchwand verwachsenen Krebses, dessen Geschwür nach außen durchgebrochen war; einmal vollzogen wir bloß eine Probelaparotomie, da die regionären Drüsen sämtlich infiltriert waren. In einem Falle zeigten sich 3 Monate nach der Probelaparotomie Symptome eines Darmverschlusses, weshalb wir am 
S. romanum einen Anus praeternaturalis herstellten; dieser Kranke starb 12 Tage nach der Operation an allgemeiner Schwäche.

Unser Radikalverfahren bestand in 2 Fällen in der Resektion des Blinddarmes. In diesen Fällen vollzog Prof. Dollinger die Resektion in einem Abschnitte. Der eine Fall bezieht sich auf einen 42 jährigen Mann, bei welchem wegen Carcinoma gelatiniforme nicht nur der Blinddarm, sondern vom Dünndarme $9 \mathrm{~cm}$ und vom Dickdarme $15 \mathrm{~cm}$. mit reseziert wurde. Kranker meldete sich nach 3 Monaten mit einem ausgebreiteten Rezidiv, diesmal begnügten wir uns mit der Enteroenteroanastomose. Patient starb 2 Monate nach dieser Operation zu Hause. In einem anderen Falle vollzogen wir die Blinddarmresektion ebenfalls an einem 42 jährigen Manne. Hier erwies sich auch die Resektion einer $52 \mathrm{~cm}$ langen Dick- und Dünndarmschlinge als notwendig. Patient meldete sich nach $\mathrm{Ab}$ lauf von 5 Jahren bei uns und war vollkommen gesund. ${ }^{1}$ )

Billroth verlor von 10 Fällen 6 (Salzersche Statistik 1892), Krönlein von 6 Fällen 3 (1900), Hochenegg (1902) von 4 Fällen 2, Wölfler (1903) von 9 Fällen 6 an operativen Komplikationen. Im Durschschnitte beträgt die Zahl der operativen Todesfälle anderseits 48,8 Proz., woneben wir auf unsere Blinddarmresektionen ohne Mortalität mit Recht hinweisen können.

B) Krebs des Colon ascendens und descendens.

Wir operierten 6 Kranke mit Dickdarmkrebs; einmal war das Colon ascendens und 5 mal das Colon descendens der Sitz des Carcinoms. Der Krebs des Colon ascendens war schon bereits exulzeriert und hatte nach Durchbruch in die Bauchhöhle eine Peritonitis hervorgerufen, wovon wir uns bei der Laparotomie überzeugten. Das Carcinom des Colon descendens war in 4 Fällen mit Ileus kompliziert, in einem Falle dagegen hatte eine Perforation in die Bauchhöhle stattgefunden. In letzterem Falle beguügten wir uns mit der Probelaparotomie. Bei den mit Ileus komplizierten Fällen griffen wir 2 mal nur palliativ ein. Die Operation bestand in der Maydlschen Kolostomie. Die Kranken starben 6 resp. 14 Stunden nach unserem Eingriffe. Wie die Obduktion erwies, bedingte den

1) Prof. Dollinger vollzog die Resektion des Blinddarmes außer den oben beschriebenen Fällen noch 5 mal wegen Carcinom und sowohl bei diesen als auch bei den wegen Tuberkulose und Fibroma Coeci ausgeführten Blinddarmresektionen, hatten wir keine operative Mortalität zu verzeichnen. 
Ileus das im Dickdarm sitzende und zusammenschrumpfende Carcinoma fibrosum; den rasch eintretenden Tod nach der Operation bewirkte der Umstand, daß die ohnehin schon sehr herabgekommenen Kranken die durch den mehrtägigen Darmverschluß bedingte septische Infektion nicht mehr zu überstehen imstande waren.

Bei 2 Fällen nahmen wir Dickdarmresektionen vor. In einem Falle saß der Krebs am Colon descendens, im anderen an der Flexura sigmoidea. Die Kanken verschieden 10 resp. 48 Stunden nach der Operation unter Kollapserscheinungen. In dem einen Falle zeigte die Sektion, daß die Drüsen des Ligamentum coli schon carcinomatös infiltriert waren und daß auch schon in der Leber 2 Metastasen vorhanden waren.

\section{Der Mastdarmkrebs.}

49 an Mastdarmkrebs Erkrankte wurden aufgenommen. Dem Alter nach verteilen sie sich wie folgt:

$$
\begin{aligned}
& \text { zwischen 24-28 Jahren . . . . . . . 2 }
\end{aligned}
$$



Dem Geschlechte nach waren es 33 Männer und 16 Frauen das Verhältnis ist also 2,06:1. Aus der Anamnese sind in einém Falle luetische Mastdarmgeschwüre zu erwähnen, an deren Stelle sich später der Krebs entwickelte.

Unsere Eingriffe sind in zwei Gruppen zu trennen: in Palliativund Radikaloperationen.

\section{a) Palliativoperationen.}

Palliativoperationen vollzogen wir in der erwähnten Zeit an 18 Kranken. In einem Falle begnügten wir uns mit der Verschorfung des Tumors. In 17 Fällen machten wir die Kolostomie, welcher wir in 10 Fällen die Exkochleation des stark gewucherten und exzulerierten Carcinoms anschlossen. Die Kolostomie vollzieht Prof. Dollinger am S romanum (Coeliocolostomia). Mit einem 2-3 Finger über den linken Spina ant. sup. medianwärts geführten Schnitte dringen wir bis zur Flexura sigmoidea vor. An der 
mesenterialen Seite machen wir mit einem stumpfen Instrumente im Mesenterium ein Loch und ziehen hier einen Gazestreifen durch, auf dem der Darm gleichsam reitend wieder in die Bauchhöhle versinkt, während ein beiläufig $10 \mathrm{~cm}$ langes Stück extraperitoneal bleibt. Nur in mit Darmverschluß komplizierten Fällen nähen wir den Darm an die Bauchwand oder an das Peritoneum unter gleichzeitigem Eröffnen des Darmlumens. Sonst warten wir bis sich zwischen dem Peritoneum und derBauchwandwunde Adhäsionen gebildet haben, welche die etwaige peritoneale Infektion beim Öffinen des Darmlumens verhindern. Mit diesem gefahrlosen Eingriffe erreichen wir, daß die bekannten Folgen des Darmverschlusses vermindert waren; die Kranken nehmen an Gewicht und Kraft zu und sind dabei schmerzfrei. Den Stuhlgang regelt man durch Darreichung milder Abführmittel, so daß der Kranke 2-3 Stunden nach Einnehmen desselben den Stuhl einmal täglich durch die künstlich erzeugte Öffnung entleert. Patient nimmt ein Bad, die Gegend des Anus praeternaturalis wird zur Verhütung eines Ekzems mit Vaselin bestrichen und der Zustand des Kranken ist ein erträglicher geworden. Operativen Tod haben wir nicht zu verzeichnen.

Unsere Kranken starben $11_{2}^{1}-19$ Monate nach der Operation. Es starben:



Im Durchschnitt trat also der Tod nach $5^{1 / 2}$ Monaten ein.

b) Radikaloperationen.

Radikaloperationen vollzog Prof. Dollinger in 6 Fällen; 3 mal am 10., 17., 29. Tage nach vorhergeganger Kolostomie; 3 mal ohne Voroperation. Das Bestehen des Krebses führten die Kranken auf 


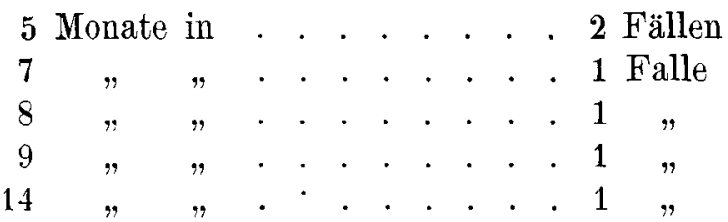

zurück. Unser operatives Verfahren war 5 mal die dorsale, 1 mal die perineale Methode. Beim dorsalen Verfahren operierten wir: 1 mal nach Rydygier, 2 mal nach Kocher, 1 mal nach Quénu und 1 mal nach Kraske.

Operative Mortalität hatten wir in einem nach Rydygier operierten Falle, wo der Mastdarm bei der Isolierung des carcinomatösen Geschwüres einriß. Die übrigen Kranken heilten sämtlich. Die operativen Todesfälle betrugen anderwärts: Rose (1889) 53 Proz., Schede und Bergmann 32 Proz., Küster 30 Proz., Garré (32 Fälle) 25 Proz., Czerny 19,4 Proz. Mit dem Fortschritt der operativen Technik wurde auch die operative Mortalität gelinger. Bei Gussenbauer 24,6 Proz., Kocher 14,2 Proz., Helferich 13,6 Proz., Czerny 11,7 Proz., Kraske 19,8 Proz., Poppert 6,22 Proz., Depage 6 Proz. Unsere operative Mortalität betrug 16,6 Proz.

Nehmen wir als Maßstab der definitiven Heilung das von Volkmann beanspruchte Triennium an, so kommen alle 6 Fälle in Betracht, bezw. da ein Kranker an operativem Tode verschied, 5 Fälle. In einem nach $\mathrm{K} 0 \mathrm{cher}$ operierten Falle trat nach 3 Monaten eine inoperables Rezidiv auf, und Kranker starb 6 Monate nach der Operation. Der nach Kraske operierte Fall starb nach 5 Monaten an einer uns unbekaunten Todesursache. Bei dem auf perinealem Wege operierten Kranken, wo der Eingriff in dem Entfernen eines keilförmigen Stückes des Sphinkters bestand, trat das lokale Rezidiv nach einem Jahre ein und der Kranke starb 14 Monate nach der Operation. Ein nach Kocher operierter Fall ist beute nach 6 Jahren rezidivfrei und war, wie er uns mitteilt, "seit der Operation niemals krank gewesen." Der nach Quenu operierte Kranke ist $4^{1 / 2}$ Jahre nach der Operation rezidivfrei.

Ziehen wir bei Beurteilung des operativen Heilerfolges den einen operativen Todesfall von den 6 Fällen ab, und beziehen die 2 Fälle, welche das Triennium schon überstanden haben, nur auf die übrigbleibenden 5, so entspricht dies 40 Proz. definitiver Heilung, auf alle 6 Fälle bezogen: 33,3 Proz.

Auf 5 Jahre lassen sich nur 4 Fälle verwerten, von denen 1 an operativem Tode, 2 an Rezidiven starben, während der eine 
genas. Den operativen Tod abgezogen entspricht dies: 33,3 Proz., auf alle 4 Fälle bezogen hingegen: 25 Proz. definitiver Heilung.

Hochenegg operierte in 89 Fällen. Hiervon leben 32 rezidivfrei, doch haben bloß 14 das Triennium erreicht, was einer Heilung in 15,72 Proz. entspricht. Garré heilte von 51 radikal Operierten $4=16,3$ Proz; die Klinik Czernys 17,3 Proz. Von Kraskes 80 Operierten leben 15, von denen 6 das Triennium erreicht haben, also 18,75 Proz., Krönlein erreichte (40 Fälle) 20 Proz., Kocher uach der Statistik von Du Pan 25,75 Proz., Gussenbauer (55 Fälle) 27,2 Proz., oder auf seine sämtlichen 72 Fälle bezogen 20,5 Proz., Depage (30 Fälle) 23 Proz., jedoch war bei letzterem die angenommene Grenze der Rezidivfreiheit 2 Jahre. Krönlein referierte im XXIX. Kongreß der „Deutschen Gesellschaft für Chirurgie" auf Grund von 11 Statistiken über 640 Fälle, eine nach 3 Jahren bestehende Rezidivfreiheit war in 14,8 Proz., d. h. in $1 / 7$ aller Fälle vorhanden.

\section{Der Peniskrebs.}

Mit Peniscarcinom gelangten 13 Kranke zur Aufnahme. Das Alter der Patienten zeigt folgende Tabelle:

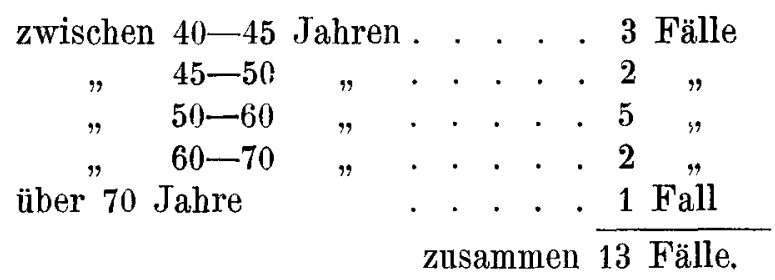

In der Anamnese wird die kongenitale Phimose häufig erwähnt, welchem Umstande besonders Cooper einen großen Wert beimißt. Das sich bei Phimose ansammelnde präputiale Sekret hält nämlich einen chronischen Reiz aufrecht. Bei uns gesellte sich der Krebs in 6 Fällen zur Phimose, also in 46,1 Proz. aller Fälle Kaufmann fand dies bei 17,7 Proz., Küttner bei 54,5 Proz., Demarquay und Hey in 75 Proz. Schuchardt stellt auch Psoriasis präputialis als häufigen Beweggrund dar; auch wir fanden in einem Falle opakfarbige Plaques auf der Eichel, welche für diese Art der Psoriasis angeblich bezeichnet sind.

In einem Falle erwähnt Patient erlittenes Trauma als den Grund des Krebses. Der Kranke besaß eine kongenitale Phimose und wahrscheinlich machte ihn das erlittene Trauma auf die schon 
bestehende Geschwulst aufmerksam. In unseren Fällen war die Größe der Tumoren von Nußform bis zum Umfange einer Mannesfaust und befanden sich sämtlich schon im exulzerierten Stadium. In 5 Fällen (38,4 Proz.) waren auch schon die Schwellkörper infiltriert und in 3 Fällen hatte sich das Carcinom per continuitatem auf die Harnröhre erstreckt und in derselben eine Fistel zustande gebracht. Die Infiltration und Anschwellung der regionären Drüsen fanden wir bei 11 Fällen (84,6 Proz.). Einmal sahen wir sogar über der Symphyse eine große Metastase und zugleich in der linken Inguinalgegend einen beweglichen Tumor von der Größe einer Mannesfaust.

Die Kranken suchten uns 4 Monate bis zwei Jahre nach Begimn ihres Leidens auf:

$$
\begin{aligned}
& \text { nach } 4 \text { Monaten in . . . . . . } 1 \text { Falle }
\end{aligned}
$$

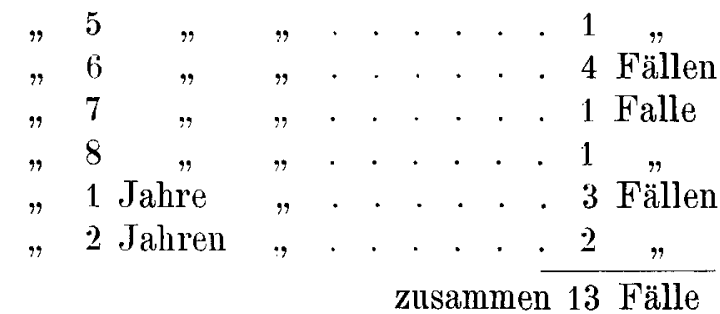

durchschnittlich also nach y Monaten.

Bei der Operation entfernten wir die inguinalen Drüsen immer und begnügten uns in jenen Fällen, wo auch schon die Corpora cavernosa infiltriert waren, nicht mit einer einfachen Amputation, sondern vollzogen die Pearce-Gouldsche Operation. Bei dieser Methode werden die Schwellkörper bis zu ihrem am Knochen haftenden Ende entfernt.

Operative Mortalität hatten wir keine. In der erwähnten Zeit operierten wir 6 Fälle. Der Krebs bestand in den operierten Fällen seit 5 Monaten bis zu einem Jahre. In 3 Fällen vollzogen wir die Amputation und in 3 Fällen vollzogen wir das PearceGouldsche Verfahren. Der weitere Verlauf ist uns bei 4 Fällen bekannt und nach 3 Amputationen und 1 Pearce-Gouldschen Operation. Von den amputierten Fällen lebt einer seit 4, einer seit 3 Jahren, während wir bei einen Fall (71 Jahre alt) an einer interkurrenten Krankheit verloren. Patient, an welchem wir die Operation nach Pearce-Gould vollzogen, lebt seit $41_{2}$ Jahren ohne Rezidive. Daher sind von 
3 Penisamputationen:

nach 3 Jahren rezidivfrei . . . 2 Fälle an interkurrenter Krankheit gestorben 1 Fall.

3 Penisexstirpationen nach Pearce-Gould:

nach 3 Jahren rezidivfrei . . . . 1 Fall unbekannt . . . . . . . . 2 Fälle.

Bruns erreichte in 59,46 Proz. eine definitive Heilung, während 40,54 Proz. an Rezidiven starben. Die Rezidiven zeigten sich in 87 Proz. aller Fälle in den 3 ersten Jahren nach der Operation und befielen meistens die regionären Drüsen.

\section{Der Krebs der Extremitäten.}

Die an den Extremitäten befindlichen bösartigen Tumoren sind meist Sarkome. In 37 Fällen von 46 hatten wir es mit einem vom Knochen oder den Weichteilen ausgehenden Sarkome zu tun, während wir den Krebs in bloß 9 Fällen diagnostizieren konnten; also war der Krebs in 19,4 Proz. aller Fälle vertreten. Auch die Erfahrungen von Bruns bestätigen, daß der Krebs der Extremitäten seltener ist. Das Alter der Kranken variierte zwischen 26 bis 91 Jahren:

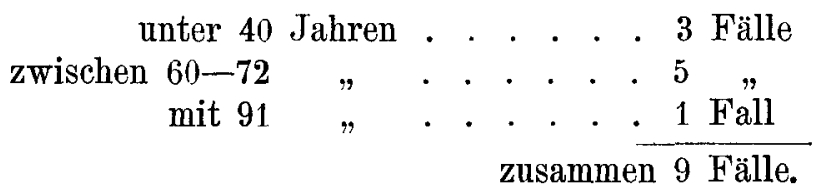

Dem Geschlechte nach waren es 5 Männer und 4 Frauen. Bei weiblichen Kranken saß der Tumor aussehließlich am Arme, was Bruns' Beobachtung zu bestätigen scheint, nach welcher die am Arme sitzenden Carcinome größtenteils bei Frauen vorkommen. Der Krebs befiel in 5 Fällen die rechte, in 4 Fällen die linke Seite und saß in 3 Fällen am rechten Arme, in 3 Fällen am linken Arme, in 2 Fällen am rechten Bein und in einem Falle an linken Bein. Der Krebs saß somit in 55,5 Proz. rechterseits, was mit Bruns' 58,7 Proz so ziemlich übereinstimmt. Die mikroskopische Untersuchung ergab in allen Fällen einen Epithelkrebs. Der Tumor war meist exulzeriert und in 6 Fällen waren auch die regionären Drüsen infiltriert. Interesse hat die Anamnese. Auch Bruns mißt den Fisteln, Narben, Geschwüren und Verrucen, chronischen 
Entzündungen Bedeutung bei, und auch wir machten die Erfahrung; daß sich der Krebs meist auf bereits krankhaft veränderten Gebieten entwickelt hatte. In einem Falle war der Oberarm eines 63jährigen Mannes seit seiner Kindheit von Psoriasis befallen, in einem anderen Falle entwickelte sich der Krebs in den Narben eines vor 26 Jahren abgelaufenen osteomyelitischem Prozesses nach einem neueren Insult. In einigen Fällen war eine Verruca der Ausgangspunkt, in 3 Fällen entwickelte sich der Prozeß in einem nach Verbrennung zurückgebliebenen Granulationsgewebe: in 2 Fällen nach 19, in 1 Falle nach 35 Jahren. Bruns betont, daß nach dem chronischen Reizzustand oder nach der anatomischen Veränderung meist 20 Jahre vergehen, ehe sich der Krebs entwickelt; auch unsere Fälle bestätigen diese Behauptung. Eigens hervorzuheben ist einer unserer Fälle, wo an verschiedenen Körperstellen kleine Krebsgeschwüre auftraten; der mikroskopische Befund war Plattenepithelkrebs.

In der erwähnten Zeit vollzogen wir 3 Radikal- und 1 Palliativoperation. Das Bestehen der Krankheit führten die Patienten von 3 Monaten bis zu 2 Jahren zurück. Bloß palliativ operierten wir in einem Falle, in welchem nach einem jauchenden handtellergroßen Carcinomgeschwür des Oberarmes anch schon die supraclavicularen Drüsen infiltriert waren. Die ausgebreitete Drüseninfiltration machte den therapeutischen Wert des Radikalverfahrens schon im vorhinein fraglich, so daß wir vom letzteren Abstand nahmen. Die Radikaloperationen beziehen sich auf folgende Fälle:

Bei einem Manus-Carcinom enukleierten wir die ganze Hand. Patient ist heute 6 Jahre nach der Operation rezidivfrei.

Bei 2 Carcinoma genu vollzogen wir die Smithsche KnieEnukleation. Der eine 91 jährige Patient starb zu Hause ohne Rezidiv an Altersschwäche, der andere ist 3 Jahre nach der Operation rezidivfrei.

Bei einem Carcinom des Unterarmes entfernten wir das krebsige Geschwür. Patient ist nach 3 Jahren rezidivfrei.

Nach 4 radikalen Operationen hatten wir also keinen operativen Tod und kein Rezidiv zu verzeichnen.

Bruns erreichte 35 Proz. definitive Heilung bei Carcinom der Extremitäten (nach Volkmana gerechnet). Wir schreiben unseren guten Erfolg lediglich dem zu, daß wir sowohl bei Amputationen, als auch bei konservativen Operationen die regionären Drüsen immer mit entfernten. 


\section{Resumé.}

Wir haben nun über die operativ erreichbaren Heilerfolge berichtet. Die Zahlen beweisen, daß es sich für den Chirurgen in hohem Maße lohnt, sich mit der Therapie des Krebses zu befassen. Um zu eruieren, welchen Erfolg wir bei der operativen Behandlung des Krebses aufweisen können, scheint es uns für richtig, die erreichten Resultate ohne Rücksicht der befallenen Regionen zu summieren und bloß die primären Krebse von den Rezidivierenden zu trennen. Wir erhalten so von unseren Resultaten eine allgemeine Übersicht. Wir gingen von den dargelegten Prinzipien aus, welche Prof. Dollinger im kgl. Ärzteverein (4. März 1905) aufstellte und welche er in seinem, in der 37. Nummer 1905 der „Deutschen Medizinischen Wochenschrift" erschienenen Artikel ausführlich begründete. Auf Grund der eingelaufenen Antworten und teilweise nach unserer eigenen Anschauung berechneten wir in wieviel Fällen die Kranken 3 resp. 5 Jahre nach der Operation rezidivfrei geblieben sind. Die 3jährige Basis war deshalb notwendig, da die Statistiken alle auf dem von Volkmann geforderten Triennium beruhen. Doch halten wir auch die Rechnung nach 5 Jahren, welche Winter im Gießener Gynäkologischen Kongreß empfahl, für zweckmäßig, da sie ein noch einwandfreieres Bild gibt, nachdem die Spätrezidiven nach dem 5. Jahre sehr selten sind.

Die Art und Weise der Berechnung unseres operativen Erfolges haben wir in der Einleitung bereits besprochen.

Bei Beurteilung des operativen Erfolges dürfen wir es nicht außer acht lassen, daß uns die Krebskranken meist in schon sehr vorgeschrittenem Stadium aufsuchen, während sie vorher alle möglichen Hausmittel versuchten, um ihr Leiden zu heilen. Die in dieser Richtung von uns angestellten Erforschungen ergaben, daß von 128 Fällen 52 sich noch nie an einen Arzt gewendet hatten, so daß 40,62 Proz. der Fälle bei uns die erste ärztliche Hilfe in Anspruch nahmen. Wir wollen noch bemerken, daß bei der Beurteilung des Erfolges gewiß auch jener Umstand in die Wagschale fällt, daß hier die Erfolge nicht eines, sondern mehrerer Operateure zusammengefaßt sind. Nachdem wir das Prinzip unserer Berechnung voransgeschickt, wollen wir die Resultate überblicken: 


\section{Primäre Krebsoperationen.}

Die dreijährige Rezidivfreiheit als Basis genommen, kommen 193 Fälle in Betracht. Der weitere Verlauf ist uns bei 150 bekannt, bei 43 unbekannt. Von den bekannten 150 Fällen sind:

$$
\begin{aligned}
& \text { nach } 3 \text { Jahren rezidivfrei . . . . } 62 \text { Fälle }=41,33 \text { Proz. } \\
& \text { innerhalb } 3 \text { Jahre rezidiviert . . . . } 71 "=47,33 \text { " } \\
& \text { an operativem Tode starben . . . . } 13 "=8,66 " \\
& \text { an interkurrenten Krankheiten starben } 4,=2,68 \text { " } \\
& 150 \text { Fälle. }
\end{aligned}
$$

Die fünfjährige Rezidivfreiheit als definitive Heilungsgrenze genommen, so kommen bloß 83 Fälle in Betracht, der weitere Verlauf ist uns bei 62 bekannt, bei 21 unbekannt.

Von diesen sind:

$$
\begin{aligned}
& \text { nach } 5 \text { Jahren rezidivfrei. . } 26 \text { Fälle }=41,93 \text { Proz. } \\
& \text { innerhalb } 5 \text { Jahre rezidiviert . } 34,=54,83 \text { " } \\
& \text { an operativem Tode gestorben } 2, \quad=3,22 \text { " } \\
& 62 \text { Fälle }
\end{aligned}
$$

\section{Rezidiv-Krebsoperationen.}

In diese Gruppe gehören jene Fälle, welche entweder nach einer auswärts oder bei uns vollzogenen Operation sich bei uns wegen Rezidiven behufs einer neueren Operation meldeten.

Die dreijährige Rezidivfreiheit gerechnet, kommen 33 Fälle in Betracht, von diesen ist der weitere Verlauf uns in 25 bekannt, unbekannt in 8 Fällen. Von den 25 bekannten Fällen sind:

$$
\begin{aligned}
& \text { nach } 3 \text { Jahren rezidivfrei . . . } 8 \text { Fälle }=32 \text { Proz. } \\
& \text { innerhalb } 3 \text { Jahren rezidiviert . } \frac{17 \mu}{25 \text { Fälle }}=68 "
\end{aligned}
$$

Die fünfjährige Rezidivfreiheit als Basis genommen, können wir 26 Fälle verwerten, von diesen hatten 18 einen bekannten und 8 einen unbekannten Verlauf. Von diesen sinà:

nach 5 Jahre rezidivfrei . . 7 Fälle $=38,88$ Proz. innerhalb う Jahre rezidiviert . $11,=61.11$, 


\section{Primär- und Rezidiv-Krebsoperationen.}

Anf drei Jahre berechnet.

Einmal operierte Krebskranke, deren weiteres Schicksal bekannt ist . . . . . . . . 150

Mehrmals operierte Krebskranke, deren weiteres Schicksal bekannt ist . . . . . . 25

Zusammen 175

Von diesen sind an Komplikationen des

Wundverlaufes gestorben . . . . . 13=7,42 Proz.

Von diesen sind an interkurrenten Krank-

heiten gestorben. . . . . . . . $4=2,28$ "

Rezidive bekamen . . . . . . . $88=50,28$ "

Rezidivfrei blieben . . . . . $\div \frac{70}{175}=40,00$ "

Auf fünf Jahre berechnet.

Einmal operierte Krebskranke, deren weiteres Schicksal bekannt ist. . . . . . . . . 62

Mehrmals operierte Krebskranke, deren weiteres Schicksal bekannt ist $\cdot \cdot \frac{\cdot}{\text { Zusammen }} 80$

Von diesen sind an Komplikationen des

Wundverlaufes gestorben . . . . $2=2,50$ Proz.

Rezidive bekamen. . . . . . . . . $45=56,25$,

Rezidivfrei blieben . . . . . . $33=41,25$ ",

Fassen wir die Dauererfolge derjenigen operierten Krebskranken, deren weiteres Schicksal wir kennen, zusammen, so sehen wir, daß von den einmal Operierten mindestens drei Jahre nach der Operation 41,33 Proz, von den mehrmals Operierten 32 Proz., mindestens fünf Jahre nach der Operation von den einmal Operierten 41,93 Proz., von den mehrmals Operierten 38,88 Proz. rezidivfrei lebten.

Nehmen wir die einmal und die mehrmals Operierten zusammen, so leben von sämtlichen Operierten rezidivfrei:

mindestens 3 Jahre nach der Operation 40,00 Proz.

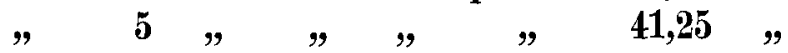


Zum Schlnsse sei es mir gestattet, meinem hochverehrten Chef und Lehrer, Herrn Prof. Dollinger, für den Arbeitsanftrag und für die Leitung während dieser Arbeit meinen verbindlichsten Dank auszusprechen.

(Der statistische Teil dieser Arbeit wurde Oktober 1904 geschlossen.)

\section{Literaturverzeichnis. ${ }^{1}$ )}

1. Dollinger, Chirurgische Methoden und klinische Jahrbücher. 3 Bände (ungarisch) 1897-1904.

2. Bergmann, Bruns, Mikulicz, Handbuch der prakt. Chirurgie.

\section{Einleitung.}

3. Kahlden, Über Carcinomrezidive. Archiv f. klin. Chir. 68, Bd. 2, 4.

4. Labhardt, Zur Frage der Dauerheilungen des Krebses. (Klinik Garré.) Beitr. z. klin. Chir. 1902, 32. Bd.

\section{Der Gesichtskrebs.}

5. Gurlt, Beiträge zur chirurgischen Statistik. Arch. f. klin. Chir., 25. Bd.

6. Heimann, Die Verbreitung der Krebserkrankung. Arch. f. klin. Chir., 53. $\mathrm{Bd}$.

\section{Der Lippenkrebs.}

7. Winiwarter, Beitr, zur Statistik der Carcinome. 1878.

8. Ebel, Znr Statistik des Carc. der Unterlippe. (Klinik Wölfler.) Beitr. z. klin. Chir., 40. Bd., 3. H.

9. Loos, Zur Statistik des Lippenkrebses auf Grund von 565 Fällen aus der v. Brunsschen Klinik. Beitr. z. klin. Chir., 27. Bd.

III. Der Mund-, Rachenhöhlen- und Wangenkrebs.

10. Pólya und $\mathrm{N}$ avratil, Untersuchungen über die Lymphbahnen der Wangenschleimhaut. Deutsche Zeitschr. f. Chir. 1902, Bd. 66, H. 1-2.

11. Pólya, Kasuistische Beiträge zur Wangenkrebsoperation. (Klinik Dollinger). (Ungarisch.) Orvosi Hetilap 1902.

\section{Der Zungenkrebs.}

12. Binder, Über 40 Fälle von Zungencarc., ihre Behandlung und Endresultate. (Klinik Krönlein.) Beitr. z. klin. Chir. 1896, 17. Bd.

13. Poirier, Traitement du cancer de la langue. Revue de Chir. 1904, Nr. 4.

V. Der Kehlkopfkrebs.

14. Sendziak, Die bösartigen Geschwülste des Kehlkopfes. 1897.

\section{Der Brustdrüsenkrebs.}

15. Ehrich, Vortrag über d. Brustdrüsenkrebs. (Klinik Trendelenburg, Madelung, Garré.) Münchner med. Wochenschr. 1905, Nr. 16.

1) Hier sollen nur die neueren Werke erwähnt werden; ein umfangreicheres Literaturverzeichnis finden wir in den folgenden Arbeiten. 
16. Schröder, Zur Dauerheilung des Brustkrebses. (Rostocker Klinik.) Beitr. z. klin. Chir. 1905, 45. Bd.

17. Mahler, Über die in der Heidelberger Klinik 1887-1897 behandelten Fälle von Carc. mammae. Beitr. z. klin. Chir., 26. Bd.

18. Schmidt, Beitrag zur Statistik der Mammacarcinome. (Klinik Braun, Göttingen.) Deutsche med. Wochenschr. 1904, Nr. 15.

19. Meiß1, Über die operative Therapie des Mammacarcinoms und deren Dauererfolge. (Klinik v. Eiselsberg.) Wiener klin. Wochenschr. 1904.

20. Gebele, Zur Statistik der Brustdrüsengeschwülste. (Klinik Angerer.) Beitr. z. klin. Chir. 1901, 29. Bd.,

21. Schwarz, Zur Statistik und Behandlung der Mammacarc. (Klinik Hacker.) Beitr. z. klin. Chir. 1905, 46. Bd, 3 H.

22. Steinthal, Zur Behandlung des Brustdrüsenkrebses. Beitr. z. klin. Chir. 1905, 47. Bd., 1. H.

23. Chavannaz, Des meilleures méthodes chirurgicales à opposer au cancer du sein. Paris 1902.

24. Guleke, Beitrag zur Statistik des Mammacarc. (Klinik v. Bergmann.) Arch. f. klin. Chir. Bd. 64, H. 3.

25. Mayo Robsen, The Lancet 1904, No. 4240.

\section{Der Magenkrebs.}

26. Chlumsky, Über die Gastroenterostomie. (Klinik v. Mikulicz.) Beitr. z. klin. Chir. 1898, 20. Bd.

27. Krönlein, Über den Verlauf des Magencarc. bei operativer und bei nichtoperativer Behandlung. Arch. f. klin. Chir. 67. Bd., 3. H.

28. Matti, Beitr. zur Chirurgie des Magenkrebses. (Klinik Kocher). Deutsche Zeitschr. f. Chir. 1905, 77. Bd,, 1-3. H.

29. Clairmont, Bericht über 258 von Prof. v. Eiselsberg ausgeführte Magenoperationen. Archiv f. klin. Chir. 1905, 76. Bd.

30. Ringel, Die Resultate der operativen Behandlung des Magencarc. Beitr. z. klin. Chir. 38. Bd., 2. H.

31. Steinthal, Erfahrungen über Gastroenterostomie. Beitr. z. klin. Chir. 1902, 34. Bd.

32. Stendel, Die in den letzten Jahren an der Czernyschen Klinik ausgeführten Magenoperationen. Beitr. z. klin. Chir. 1899, 23. Bd.

33. Stich, Beiträge zur Magenchirurgie. (Klinik Garré.) Beitr. z. klin. Chir. 40. Bd., 2. $\mathrm{H}$.

34. Schönholzer, Die Chirurgie des Magenkrebses an der Krönleinschen Klinik in den Jahren 1881-1902. Beitr. z. klin. Chir., 39. Bd., 1. H.

35̄. Brüning, Beitr. zur Frage der Resektion des carcinomatösen Magens. (Klinik Poppert.) Deutsche Zeitschr. f. Chir. 1905, 80. Bd., 1.-2. H.

36. Neck, Zur Technik der Magenausspülung. (Krankenhaus in Chemnitz.) Zentralbl. f. Chir. 1902, Nr. 52.

37. Guinard, La cure chirurgicale du cancer de l'estomac. Paris 1898.

\section{Der Dïnn- und Dickdarmkrebs.}

38. Hochenegg, Resultate bei oper. Behandlung carcinomatöser Dickdarmgeschwülste. Archiv f. klin. Chir., 68. Bd., 1. H. 
39. Mikulicz, Chirurgische Erfahrungen über d. Darmcarcinom. Archiv f, klin. Chir., 69. Bd. $1 \mathrm{H}$.

40. Schl of fer, Zur operativen Behandlung des Dickdarmcarc. (Klinik Wöl fler.) Beitr. z. klin. Chir. 1903, 38. Bd.

41. Zimmermann, Über Operation und Erfolge der Dickdarmresektion wegen Carcinom. (Klinik Krönlein.) Beitr. z. klin. Chir. 1900, 28. Bd.

42. Körte, Erfahrungen über das Dickdarmcarcinom. Berliner klin. Wochenschrift 1899.

43. Czerny und Rindfleisch, Operationen am.Magen und Darm. Beitr. z. klin. Chir. 1892.

44. Salzer, Beiträge zur Pathologie und chirurg. Therapie chron. Coecumerkrankungen. Arch. f. klin. Chir., X. Bd.

45. König, Die Operationen am Darm bei Geschwülsten. XIX. Chir.-Kongr. 1890.

\section{Der Mastdarmkrebs.}

46. Christen. Beiträge zur Statistik und operativen Behandlung des Mastdarmkrebses. (Klinik Krönlein). Beitr. z. klin. Chír., 27. Bd.

47. Depage, Résultats éloignés de la résection du rectum pour cancer. XVII. Congrès français de Chirurgie. Paris 1904.

48. Hackmann, Zur Statistik und operativen Behandlung der Rektumearcinome. (Klinik Gussenbauer.) Arbeiten aus dem Gebiete der klin. Chir. Wien 1903.

49. J affé, Indikation und Prognose der Operation des Mastdarmkrebses. Arch. f. klin. Chir. 1902, 67. Bd., 1. H.

50. Lieblein, Zur Statistik und Technik der Radikaloperation des Mastdarmkrebses. (Klinik Wölfler.) Beitr. z. klin. Chir. 1902, 33. Bd.

51. Schneider, Die Behandlung des Rektumcarcinoms. (Klinik Garré.) Beitr. z. klin. Chir. 1900, 26. Bd.

52. Richter, Über die auf der Gießener Klinik erzielten Erfolge bei der Radikaloperation des Mastdarmcarcinoms. (Klinik Poppert.) Deutsche Zeitschr. f. Chir. 1906, 81. Bd.

53. D u Pan, Contribution à l'étude du traitement du cancer du rectum. (Clinique du Kocher.) Thèse de Berne. 1905.

\section{Der Peniskrebs.}

54. Küttner, Über das Peniscarcinom. (Klinik Bruns.) Beitr. z. klin Chir. 1900.

\section{Der Krebs der Extremitäten.}

55. Brunn, Über den primären Krebs der Extremitäten. (Klinik Bruns.) Beitr. z. klin. Chir. 1903, 37. Bd.

\section{Zusammenfassung.}

50. Dollinger, Die Dauererfolge der operativen Behandlung des Krebses in der I. chirurg. Universitätsklinik in Budapest. Deutsche med. Wochenschr. $1905, \mathrm{Nr}, 37$.

57. Léjars, Utilité et limites ne l'action chirurg. dans le cancer. La Sémaine Médicale 1903, Nr. 50. 